\title{
Effects of beam wobbling and target rotation on the target temperature in experiments with intense heavy ion beams
}

\author{
R. N. Sagaidak®* \\ Flerov Laboratory of Nuclear Reactions, Joint Institute for Nuclear Research, \\ J.-Curie 6, 141980 Dubna, Moscow Region, Russia
}

(Received 29 April 2021; accepted 7 July 2021; published 12 August 2021)

\begin{abstract}
Effects of beam wobbling and target rotation on reducing target temperature are quantitatively considered with simulations and calculations. These manipulations with the beam and target reduce sharpness in the beam-density distribution, making it quasiuniform on the target surface. A uniform beam density is essential in prolonged experiments on the synthesis of superheavy nuclei using intense heavy-ion beams and actinide targets. The heavy-ion beam energy partially absorbed by the target and target backing heats them and transfers warmth to the surrounding by different means. The target temperature was initially considered for a stationary target using notions of heat transfer due to the thermal conductivity, radiation emission, and heat removal to dilute gas surrounding the target. The effects of the beam width, the amplitude of the wobbler, and the rotating target velocity on the beamdensity distribution across the target surface and, consequently, on its temperature are further estimated with the same notions.
\end{abstract}

DOI: 10.1103/PhysRevAccelBeams.24.083001

\section{INTRODUCTION}

Complete fusion reactions induced by the ${ }^{48} \mathrm{Ca}$ projectile on actinide target nuclei allowed one to synthesize superheavy nuclei (SHN) with $112 \leq Z \leq 118$ [1]. In the Dubna discovery experiments, the ${ }^{48} \mathrm{Ca}$ beam delivered to the target had the intensity of $\sim 1 \mathrm{p} \mu \mathrm{A}$ that allowed one to obtain several atoms of SHN per month at the production cross section of a few pb and the efficiency provided by the Dubna gas-filled recoil separator (DGFRS) used in experiments [1,2]. In these experiments, the actinide targets of $0.3-0.8 \mathrm{mg} / \mathrm{cm}^{2}$ of thickness were deposited on Ti backing foils of $0.71-0.73 \mathrm{mg} / \mathrm{cm}^{2}$ of thickness and maintained on a rotating wheel. The target wheel placed in the atmosphere of hydrogen at the pressure of 1 Torr withstood the beam's load in long-term experiments. A beam dose exceeding $10^{19}$ particles was usually collected for a beam time [1].

The detailed study of SHN properties and experiments on the synthesis of heavier SHN with $Z>118$ need more intense heavy-ion (HI) beams than those used before. Such beams are now provided by the high-current cyclotron DC280 recently commissioned at the Flerov Laboratory

\footnotetext{
*sagaidak@jinr.ru
}

Published by the American Physical Society under the terms of the Creative Commons Attribution 4.0 International license. Further distribution of this work must maintain attribution to the author(s) and the published article's title, journal citation, and DOI. of Nuclear Reactions (FLNR) of JINR. The intensity of the ${ }^{48} \mathrm{Ca}$ beam delivered to a physical target is close to the expected one [3]. A power density released inside the targets and target backing amounts from several tens to several hundreds of $\mathrm{W} / \mathrm{cm}^{2}$. The synthesis of SHN with $Z>118$ implies heavier beam particles such as ${ }^{50} \mathrm{Ti}$ and ${ }^{54} \mathrm{Cr}$. For SHN formed in the fusion-evaporation reactions with these projectiles, one may expect the production cross sections of $\sim 0.05 \mathrm{pb}$ [4]. To observe several events of these SHN, one should collect the beam dose exceeding $10^{20}$ particles. This dose can be provided at the intensity of $5-10 \mathrm{p} \mu \mathrm{A}$ or $(3.12-6.24) \times 10^{13} \mathrm{p} / \mathrm{s}$ for a reasonable beam time.

High heating powers released inside a target as well as high-beam doses reduce its durability. One would think that the target and its backing are mainly degraded due to the sputtering of their atoms, radiation damages of their crystal lattices, and thermal loads. All the processes are nonindependent, but they can be evaluated by treating them separately [5]. The target temperature is determined by the heating power released inside the target and backing and conditions of heat removal from a hot spot formed by a HI beam. In general, this heat can be removed due to the thermal conductivity of the target and backing, heat radiation emitted from them, and heat transfer to gas in the case of its presence [6-9]. Pulse heating is one of the ways to reduce thermal load. In the case of a continuous beam, a pulsing regime can be realized with target rotation.

Rotating target wheels of different constructions are used at the laboratories, where experiments with intense 
HI beams are carried out. The size of a target wheel mainly depends on the availability of target materials, considering that target thickness is within $0.4-0.7 \mathrm{mg} / \mathrm{cm}^{2}$ for experiments with recoil separators. The width of the target annulus usually corresponds to $0.8-1.2 \mathrm{~cm}$. Thus, in the case of experiments with available low-melting-point target materials $(\mathrm{Pb}, \mathrm{Bi})$, wheels with rather sizeable central radius $R_{c}$ of $15 \mathrm{~cm} \mathrm{[10],} 15.5 \mathrm{~cm} \mathrm{[11],} \mathrm{and} 33.5 \mathrm{~cm}$ [12] were used. For experiments with radioactive actinides, target wheels with smaller $R_{c}$ of $6 \mathrm{~cm} \mathrm{[1],} 1.675 \mathrm{~cm}$ [13], and $5 \mathrm{~cm}$ [14] were exploited. The velocity of target wheels increased over time. For example, in SHIP experiments with $\mathrm{Pb}$ and $\mathrm{Bi}$ targets, the velocity of the wheel was increased from $375 \mathrm{rpm}$ initially used [11] to $1332 \mathrm{rpm}$ set later [15]. Similar evolution from 600 to $3000 \mathrm{rpm}$ took place in experiments carried out with GARIS [10]. In TASCA experiments with actinide targets, the velocity was also increased from $2000 \mathrm{rpm}$ for a small wheel [13] to $2249 \mathrm{rpm}$ for a larger one [14]. In contrast to that, DGFRS experiments were carried out with the same target wheel of a $6 \mathrm{~cm}$ radius and at the same wheel velocity of $2000 \mathrm{rpm}$ [1]. One has to mention that rotation of target wheels used in GSI experiments is synchronized with the macrostructure of the UNILAC beam [11,13-15].

Calculations show that increasing the target wheel radius and rotation velocity allows one to reduce the average temperature of the target and the difference between its maximal and minimal values $[8,12,16]$. These calculations were performed with the uniform beam-density distribution throughout the beam spot area. At the same time, in experiments, bidimensional quasi-Gaussian distributions are usually accessible in a plane perpendicular to the beam direction. Infrared snapshots of rotating targets [7] demonstrate traces left by a HI beam on the target surface, which reflect nonuniform beam-density distributions along the target width annulus. In general, a beam profile has a bidimensional Gaussian-like shape with half-widths depending on the beam tuning. This shape may vary during the irradiation of a target in a prolonged experiment. Beam wobbling was applied in intense $\mathrm{HI}$ beam experiments with a stationary target as early as 1974 [17], making a quasiuniform beam-density distribution. In the DGFRS experiments [1], beam wobbling in the direction perpendicular to the target rotation was used to spread the high-intensity ${ }^{48} \mathrm{Ca}$ beam within a whole target annulus.

In the present work, an attempt is made to obtain quantitative estimates of the effects of target rotation and beam wobbling on the beam-density distribution across a target area. In the next section, the target temperature is considered for a stationary target and uniform and nonuniform beam-density distribution. In Sec. III, the effects of target rotation and beam wobbling on the beam-density distribution across a target surface and target temperature are presented. The last section summarizes the efforts undertaken in the present work.

\section{STATIONARY TARGET TEMPERATURE}

\section{A. Assumptions and beam-density distribution}

The importance of a uniform beam distribution, though seemingly evident, can be confirmed quantitatively with the calculation of a temperature distribution across a target area. Thermal diffusivity values allow one to estimate a time of heat diffusion away from the beam spot of radius $r_{b}$ as $\tau_{\text {thd }}=\pi r_{b}^{2} / \alpha_{\text {thd }}$. According to the recent study [18], for Ti, $\alpha_{\text {thd }}$ drops from 9.3 to $6.4 \mathrm{~mm}^{2} / \mathrm{s}$ with increasing temperature from 20 to $800{ }^{\circ} \mathrm{C}$ and then rises to $7.9 \mathrm{~mm}^{2} / \mathrm{s}$ at $1008^{\circ} \mathrm{C}$ (hereinafter the properties of pure Ti are used for the estimates). For $\mathrm{UO}_{2}$, according to Refs. [19,20], $\alpha_{\text {thd }}$ drops from 3 to $0.6 \mathrm{~mm}^{2} / \mathrm{s}$ with increasing temperature from 20 to $1500{ }^{\circ} \mathrm{C}$. For $r_{b}=r_{t} / 2$ and target radius $r_{t}=0.5 \mathrm{~cm}$, one can obtain $\tau_{\text {thd }}$ in the region of 2.1-3.1 s and 6.5-33 s for $\mathrm{Ti}$ and $\mathrm{UO}_{2}$, respectively. Rather large times for the temperature (heat) propagation allow one to consider the effect of a nonuniform density distribution across a target and target backing area. Such consideration can be done for a steadystate regime at a constant beam intensity and assuming a negligible heat transfer between neighbor annuluses, into which a circle target may be partitioned.

In general, the bidimensional Gaussian beam-density distribution can describe the beam spot spread in $x$ - and $y$-direction with standard deviations $\sigma_{x}$ and $\sigma_{y}$, respectively. In a simple case, when a correlation coefficient is equal to zero and $\sigma_{x}=\sigma_{y}=\sigma_{b}$, this distribution can be written as

$$
f(r)=1 /\left(2 \pi \sigma_{b}^{2}\right) \exp \left(-0.5 r^{2} / \sigma_{b}^{2}\right)
$$

where $r=\sqrt{x^{2}+y^{2}}$ is the deviation from the beam (target) center. Partitioning the beam spot into the annuluses of an equal area, one can obtain the ratios of the beam densities according to Eq. (1) and those corresponding to the uniform distribution. It was done with Monte Carlo (MC) simulations and the results are shown in Fig. 1.

The values of beam losses $\varepsilon_{\text {loss }}$ for transmission through the target of a $0.5 \mathrm{~cm}$ radius are indicated in the figure. They show that the beam tuning corresponding to $\sigma_{b}>0.2 \mathrm{~cm}$ leads to significant beam losses. These values will be further compared with similar ones obtained for the rotating target and beam wobbling.

\section{B. Heat removal from target}

As was mentioned above, heating power generated inside the target and target backing can be removed due to the thermal conductivity of the target and backing, radiation emitted from them, and the conductivity of gas or heat transfer to gas molecules [6-9]. Below, these processes are considered for the uniform beam-density distribution throughout a target area. 


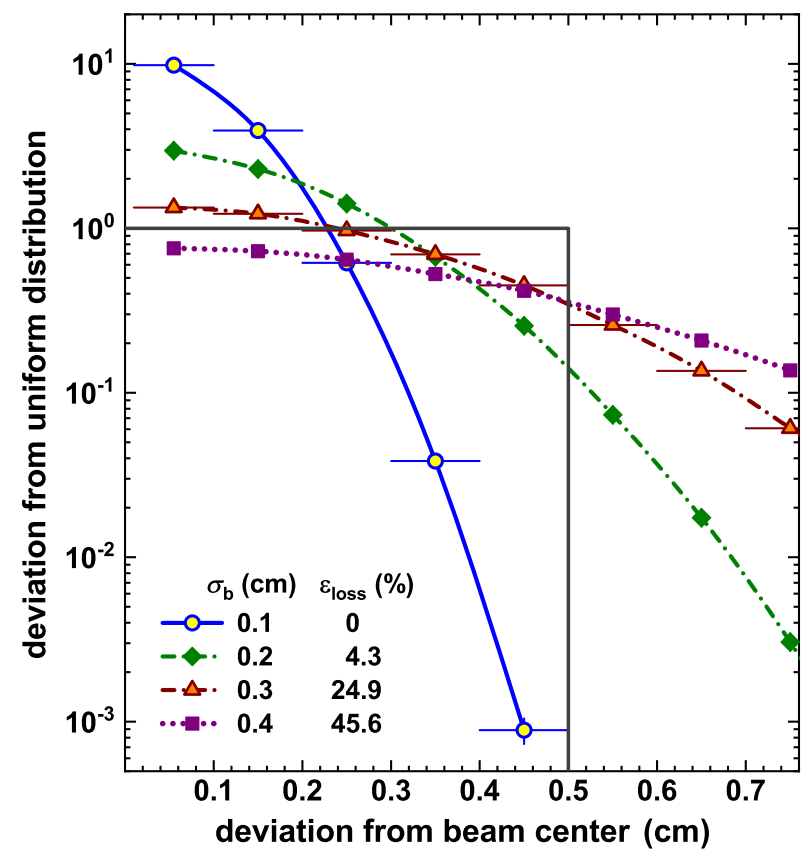

FIG. 1. The ratios of the beam densities according to Eq. (1) at different $\sigma_{b}$ values and respective values for the uniform distribution as obtained with Monte Carlo simulations. Horizontal bars for $\sigma_{b}=0.1$ and $0.3 \mathrm{~cm}$ correspond to the widths of annuluses partitioning the target of a $0.5 \mathrm{~cm}$ radius. Beam loss values $\varepsilon_{\text {loss }}$ are indicated for the respective $\sigma_{b}$.

\section{Thermal conductivity of target and backing}

For a stationary target with radius $r_{t}$ at temperature $T_{s}$, which is under surrounding temperature $T_{0}$, a power removing due to the thermal conductivity can be estimated, according to $[21,22]$, as

$$
Q_{\mathrm{cnd}}=2 \pi \lambda D\left(T_{s}-T_{0}\right) / \ln \left(r_{t} / r_{b}\right)
$$

where $\lambda$ is the coefficient of thermal conductivity, $D$ is the thickness of the target or backing foil in corresponding units, and $T_{0}=293.15 \mathrm{~K}$ is the surrounding temperature of the target frame. Note that Eq. (2) may estimate only the upper limit of heat transfer. The heat transfer is actually determined by respective transferring to the frame which is in contact with the backing foil edges clamped between two parts of the target frame. This way of heat removal leads to much smaller values of transferred heating power than those obtained with idealized Eq. (2). Heat exchange between the target itself and target backing was not considered, bearing in mind their small thicknesses and assuming a good contact between them. Equation (2) can only be used for the approximate estimates of upper limits of heat removal from a stationary target due to the thermal conductivity of the target itself and its backing.

Here and throughout the present work, temperature dependencies of parameters determining a heat transfer

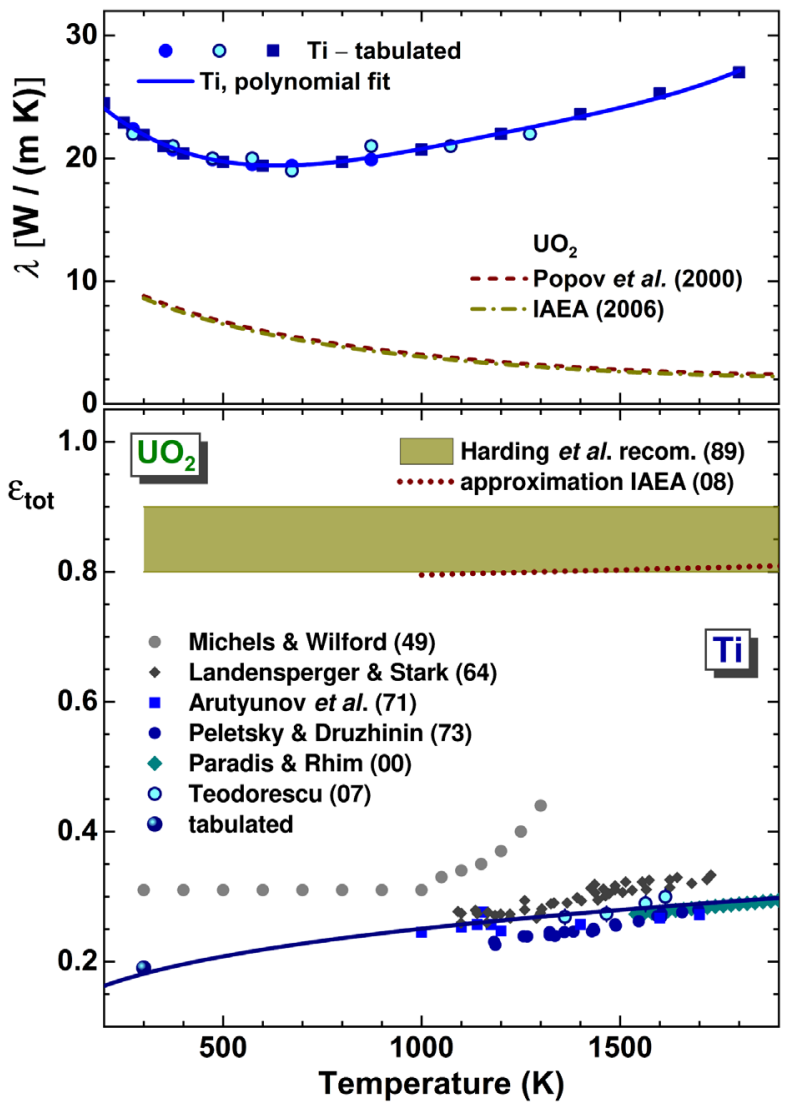

FIG. 2. Data on thermal conductivity coefficients $\lambda$ for $\mathrm{Ti}$ (symbols) [22-24] and for $\mathrm{UO}_{2}$ approximations (lines) [19,25] are shown as functions of temperature (upper panel). A solid line shows the result of the polynomial fit to the Ti data. In the bottom panel, the available data on total hemispherical emissivity $\varepsilon_{\text {tot }}$ for Ti (symbols) [26-32] together with the $a T^{b}$ function fitting the data (solid line) and approximations for $\mathrm{UO}_{2}$ (dotted line and filled area) $[33,34]$ are shown.

were used in respective estimates. In Fig. 2, a polynomial approximation to the tabulated $\lambda$ values for $\mathrm{Ti}$ [22-24] in the region of $200-1900 \mathrm{~K}$ is shown. Ti foils of the $1.5-2.5 \mu \mathrm{m}$ of thickness are preferentially used in the highintensity HI experiments with actinide targets [1,12-14] due to their durability. For $\lambda$ data of $\mathrm{UO}_{2}$, the respective approximations, corresponding to the $100 \% \mathrm{UO}_{2}$ density [19,25], are also shown in Fig. 2.

In Fig. 3, the estimates of heating power removed due to the thermal conductivity of the Ti-backing foil and $\mathrm{UO}_{2}$ target of respective thicknesses are shown as functions of temperature. These correspond to the uniform beam-density distribution within $r_{b}=0.5 \mathrm{~cm}$. In the application of Eq. (2), $r_{t}=0.7 \mathrm{~cm}$ for the backing and $r_{t}=0.55 \mathrm{~cm}$ for the target were used. The estimate with the last value is a conditional one since, in the case of a stationary target, the size of the "cool" target frame is usually larger than the target itself. With these assumptions, heat removal from the backing is $3-8$ times greater than the one from the target. 


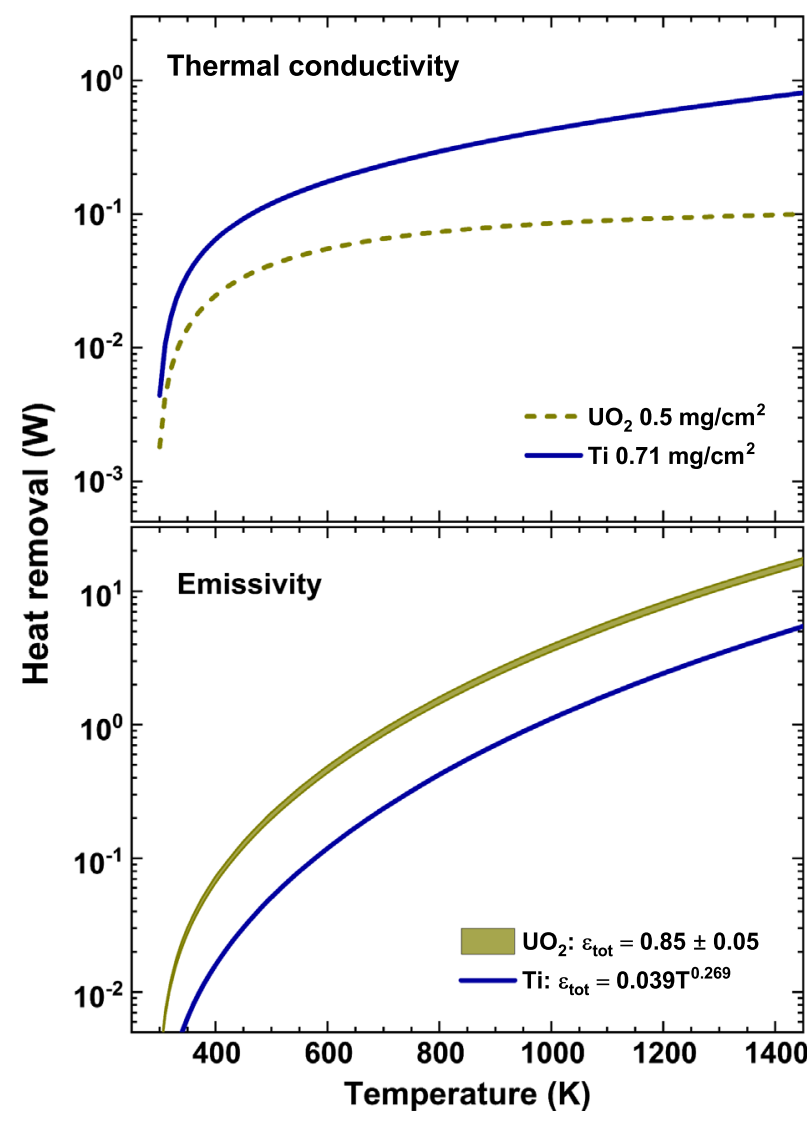

FIG. 3. Heating powers removed due to the $\mathrm{UO}_{2}$ target and $\mathrm{Ti}$ backing thermal conductivity are shown as functions of temperature in the upper panel (lines). In the bottom panel, similar heating powers removed by radiation emitted from the target and backing surfaces are shown by filled area and line, respectively. See details in the text.

\section{Emissivity of target and backing}

Heating power removed due to radiation emitted from the target (backing) surface is estimated by the StefanBoltzmann law as

$$
Q_{\mathrm{rad}}=\sigma_{\mathrm{SB}} \varepsilon_{\mathrm{tot}} F\left(T_{s}^{4}-T_{0}^{4}\right),
$$

where $\sigma_{\mathrm{SB}}$ is the Stefan-Boltzmann constant, $\varepsilon_{\text {tot }}$ is the total hemispherical emissivity, and $F$ is the target (backing) area. In Fig. 2, the available data on $\varepsilon_{\text {tot }}$ for Ti [26-32] and approximations for $\mathrm{UO}_{2}[33,34]$ are shown. Further, the heating power removed from the Ti target backing was estimated with $\varepsilon_{\text {tot }}$ values obtained using the $a T^{b}$ function fitting the data [27-32]. Fitted parameters $a=0.039 \pm$ 0.010 and $b=0.269 \pm 0.036$ were obtained as the result of the fit. For the estimates of the heating power removed from the $\mathrm{UO}_{2}$ target, $\varepsilon_{\text {tot }}=0.85 \pm 0.05$ recommended in Ref. [33] was used.

In Fig. 3, the estimates of heating power removed due to the radiation emitted from the Ti-backing foil and $\mathrm{UO}_{2}$ target are shown as functions of the temperature for the uniform beam-density distribution and $r_{b}=r_{t}=0.5 \mathrm{~cm}$. As one can see, heat removal from the target surface is 3-5 times greater than the one from the backing due to the lower emissivity of the latter.

\section{Heat removal to gas}

The estimate of heating power removed from a hot target by ambient gas is not apparent. Different approaches were used to estimate the heating power removed by rarefied gas surrounding the target [7-9].

Cagarda considered the process of heat removal to rarefied gas in the framework of the kinetic theory of gases [7]. A final expression obtained by him is as follows:

$$
Q_{\mathrm{gas}}^{\mathrm{Cag}}=\frac{3 \bar{v} \alpha p F}{8 T_{\mathrm{gas}}}\left(T_{s}-T_{\mathrm{gas}}\right),
$$

where $p$ is the gas pressure, $\bar{v}$ is the mean molecular velocity, $T_{s}$ and $T_{\text {gas }}$ are the temperatures of solid surface and gas, respectively, and $\alpha$ is the accommodation coefficient. The last is treated as a portion of the energy carried away by gas molecules from a hot solid surface. Its value depends on many factors and is varied within $0<\alpha<1$. An empirical formula describing the dependence of $\alpha\left(T_{s}\right)$ was proposed in Ref. [35], which considers molecular masses of gas and solid. Verification of this formula for $\mathrm{H}_{2}$ and $\mathrm{He}$ gases was performed using the available experimental data on $\alpha$ [36-47], including those used in Ref. [35]. The results are shown in Fig. 4 for the specified solid surfaces.

As one can see in the figure, the data follow a general trend corresponding to the drop of the $\alpha$ value with the solid surface temperature. Simultaneously, some exceptions occur for the data obtained in experiments with $\mathrm{H}_{2}$ on Pt $[41,42,47]$. Thus, the data [41] below $600 \mathrm{~K}$ remarkably deviate from similar ones [42,47] obtained later and from the calculation with the SY formula [35] (see Fig. 4). The $\alpha$ value obtained for $\mathrm{He}$ on $\mathrm{Al}$ [39] (not shown in the figure) significantly exceeds those obtained in Ref. [44]. The SY scaling reproduces the data for $\mathrm{H}_{2}$ on the "light" surfaces (graphite, stainless steel, and $\mathrm{SiO}_{2}$ ) poorly, as one can see in the figure. For further use, the $\mathrm{H}_{2}$ data were separately considered for the light $\left(\mathrm{C}-\mathrm{SiO}_{2}\right)$ and "heavy" $(\mathrm{Pt})$ surfaces, whereas the He data were treated as a whole. The sets of the data selected in this way were fitted with exponent $\alpha=a \exp \left(-b T_{s}\right)$, where $a$ and $b$ are fitting parameters. For the weighted fitting procedure, $10 \%$ relative errors were assigned to all the data points, except those, for which the absolute errors in the $\alpha$ values were originally indicated $[38,40,47]$. As the results of these fits, $a$ and $b$ parameter values are listed in Table I.

With the accommodation coefficients thus obtained and the use of Eq. (4) with $T_{\text {gas }}=\left(T_{s}+T_{0}\right) / 2$ and the expression for mean molecular velocity $\bar{v}$ : 


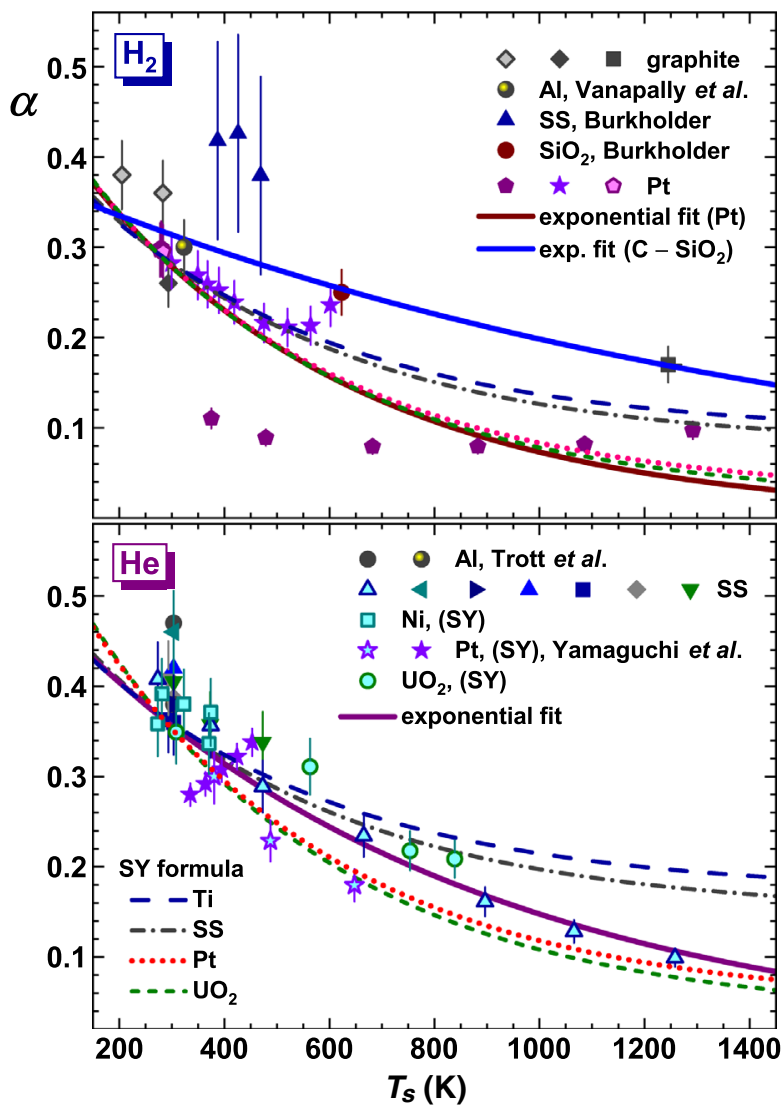

FIG. 4. Data on accommodation coefficients for $\mathrm{H}_{2}$ on graphite [36-38], Al [39], stainless steel (SS) [40], $\mathrm{SiO}_{2}$ [40], and Pt [41-43] (upper panel), and those for He on Al [44], SS [35,44-46], Ni [35], Pt [35,47], and $\mathrm{UO}_{2}$ [35] (bottom panel) are shown by different symbols (He data used in Ref. [35] are marked as [SY]). Respective lines show calculations with the empirical SY formula [35] for specified solid surfaces and the results of exponential function fitting the data.

$$
\bar{v}=\sqrt{8 R T_{\mathrm{gas}} /(\pi M)},
$$

where $R$ is the gas constant, and $M$ is the molar mass of gas, a power removed by the gas surrounding the target can be estimated. The results of these estimates for $\mathrm{H}_{2}$ and $\mathrm{He}$ rarefied gases at $p=1$ Torr are shown in Fig. 5.

Earlier, in the framework of the kinetic theory of gases, Knudsen derived a similar expression for heating power transferred from a hot surface to a cold gas [48], which can be written as

TABLE I. Fitting parameters $a$ and $b$ for the exponent $\alpha\left(T_{s}\right)=$ $a \exp \left(-b T_{s}\right)$ reproducing the data shown in Fig. 4.

\begin{tabular}{lcc}
\hline \hline Data set & $a$ & $b \times 10^{4}$ \\
\hline $\mathrm{H}_{2}$ on $\mathrm{C}-\mathrm{SiO}_{2}$ & $0.382 \pm 0.035$ & $6.56 \pm 1.63$ \\
$\mathrm{H}_{2}$ on $\mathrm{Pt}$ & $0.494 \pm 0.080$ & $19.1 \pm 2.9$ \\
$\mathrm{He}$ on $\mathrm{Al}-\mathrm{UO}_{2}$ & $0.520 \pm 0.026$ & $12.6 \pm 1.0$ \\
\hline \hline
\end{tabular}

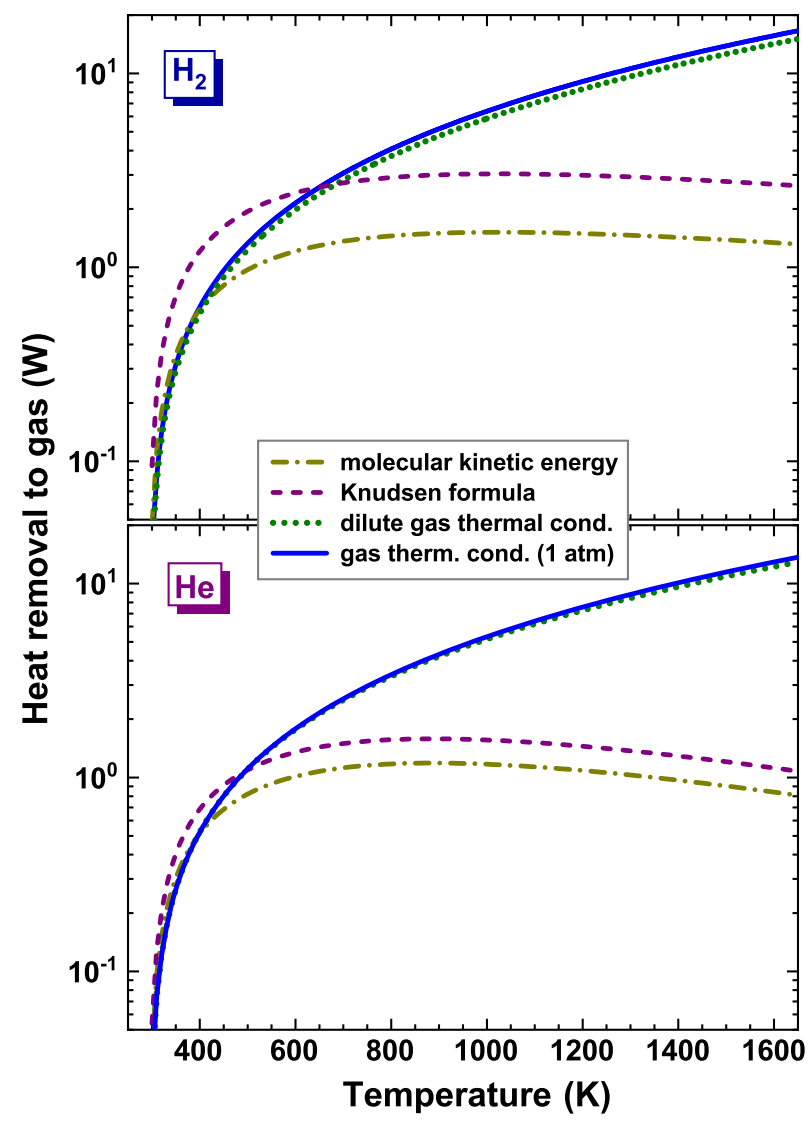

FIG. 5. Heating powers removed from the $\mathrm{UO}_{2}$ target and $\mathrm{Ti}$ backing $\left(r_{t}=0.5 \mathrm{~cm}\right)$ by rarefied $\mathrm{H}_{2}$ and $\mathrm{He}$ gases as functions of surface temperature are shown in the upper panel and bottom panel, respectively. Estimates dealing with the gas molecular kinetic energy considerations, corresponding to Eq. (4) and Knudsen's formula of Eq. (6), along with the thermal conductivity, corresponding to Eq. (11) and the approximation [52], are shown by respective lines.

$$
Q_{\mathrm{gas}}^{\mathrm{Kn}}=\frac{\gamma+1}{\gamma-1}\left(\frac{R}{8 \pi M T_{\mathrm{gas}}}\right)^{1 / 2} \alpha p F\left(T_{s}-T_{\mathrm{gas}}\right),
$$

where $\gamma$ is the ratio of specific heat capacities equal to $5 / 3$ for monatomic gases $(\mathrm{He})$ and $7 / 5$ for diatomic gases $\left(\mathrm{H}_{2}\right)$. Equation (6) differs from Eq. (4) used with Eq. (5) by a factor of $(\gamma+1) /[3(\gamma-1)]$. The results of Eq. (6) application for heating powers removed by $\mathrm{He}$ and $\mathrm{H}_{2}$ gases at $p=1$ Torr are shown in Fig. 5. In these estimates, the approximated accommodation coefficients with the parameters listed in Table I were used. Note that uncertainties in both these estimates are mainly determined by the uncertainties in the $\alpha$ values obtained with the data fit (see Fig. 4 and Table I). Their relative values increase from $6 \%$ to $16 \%$ for $\mathrm{He}$, from $10 \%$ to $26 \%$ for $\mathrm{H}_{2}$ on $\mathrm{Ti}$, and from $18 \%$ to $46 \%$ for $\mathrm{H}_{2}$ on $\mathrm{UO}_{2}$, with the increase in the temperature from 300 to $1500 \mathrm{~K}$.

According to the kinetic theory of gases, a mean free path of gas molecules corresponds to 


$$
\bar{l}=k_{\mathrm{B}} T_{\mathrm{gas}} /\left(\sqrt{2} \pi d^{2} p\right)
$$

where $k_{\mathrm{B}}$ is the Boltzmann constant and $d$ is the kinetic diameter of a molecule. At $p=1$ Torr, $T=T_{0}$, and assuming that $d=2.827$ and $2.551 \AA$ for $\mathrm{H}_{2}$ and $\mathrm{He}$ gases, respectively [23], we obtain $\bar{l}=85.5$ and $105 \mu \mathrm{m}$ for $\mathrm{H}_{2}$ and $\mathrm{He}$, respectively. The characteristic length between the target and surrounding cool surfaces is not determined in our case $(5 \lesssim L \lesssim 25 \mathrm{~mm}$ ), and the respective Knudsen number $K_{n}=\bar{l} / L$ is varied within $0.004 \lesssim L \lesssim 0.016$. This region of $K_{n}$ is close to the continuum regime. Thus, there is some reason to consider heat removal by gas regarding its bulk thermal conductivity. It was done in Ref. [9], assuming a constant value for the coefficient of thermal conductivity $\lambda_{\text {gas }}=0.2 \mathrm{~W} /(\mathrm{mK})$ for $\mathrm{H}_{2}$. This value corresponds to the temperature of $350 \mathrm{~K}$ and pressure of $1 \mathrm{~atm}[22,23,49]$. At the same time, in several experiments connected with the measurements of the heat conductivity of gases confined to a gap between hot and cold surfaces, a smooth drop in measured values determined by the heat conductivity of gas was observed with a decrease in gas pressure [38-40,44,45,47].

One can take into account gas pressure with a simple relationship using $K_{n}$ (see, for example, Ref. [50] and references therein):

$$
\lambda_{\text {gas }}=\lambda_{\text {gas }}^{0} /\left(1+2 \beta K_{n}\right),
$$

where $K_{n}$ is determined by Eq. (7) and $L, \lambda_{\text {gas }}^{0}$ is the bulk value of the coefficient of thermal conductivity (corresponding to standard temperature and pressure), and $\beta$ is the coefficient characterizing the molecule-wall collisionenergy transfer efficiency $(1.5 \leq \beta \leq 2.0)$, which is thus different from accommodation coefficient $\alpha$ considered above. Bearing in mind uncertainties in the $K_{n}$, heat transfer can be estimated with the respective uncertainty exceeding a factor 5 using Eq. (8).

More definitely, thermal conductivity for dilute gases can be considered in the framework of kinetic theory with $\lambda_{\text {gas }}$ expressed through gas viscosity $\mu$, as described in some books (see, for instance, Ref. [23]):

$$
\lambda_{\text {gas }}=\left(\frac{9 \gamma-5}{4 \gamma}\right) \mu c_{p},
$$

where $c_{p}$ is heat capacity at constant pressure and $\mu$ is gas viscosity, which is calculated as

$$
\mu=2.6693 \times 10^{-6}\left(M T_{\mathrm{gas}}\right)^{1 / 2} /\left(d^{2} \Omega_{\mu}\right),
$$

where $d$ is the effective collision diameter (in $\AA$ ) and $\Omega_{\mu}$ is the collision integral (dimensionless value). The last is the tabulated function of $\left(k_{B} T_{\text {gas }} / \epsilon\right)$, in which $\left(\epsilon / k_{B}\right)$ is a constant value for a specified gas, and $\epsilon$ corresponds to the
Lennard-Jones potential well depth (see Tables 11.2 and 11.3 in Ref. [23]). Thus using Eqs. (9) and (10) and the data for $\Omega_{\mu}, \lambda_{\text {gas }}$ can be estimated. At high temperatures, these values are somewhat lower than the tabulated data $[22,23,51]$ and approximations [49,52] for $\lambda_{\text {gas }}$ at the atmospheric pressure, as shown in Fig. 6.

With $\lambda_{\text {gas }}$ values, one can estimate heating power removed due to gas conductivity $Q_{\mathrm{gas}}^{\mathrm{cnd}}$, using shape factor $S$ determined by heat transfer geometry. In our case, this geometry may be approximated by a hot disk of radius $r_{t}$ at temperature $T_{s}$, which is surrounded by the conductive gas medium transferring heat to an infinite cold surface at temperature $T_{0}$. The cold surface is arranged parallel to the disk at distance $L$ from it. The exact solution of the respective thermal conductivity equation is known as $S_{0}=4 r_{t} \lambda_{\text {gas }}$ if $L=0$, and $S_{\infty}=8 r_{t} \lambda_{\text {gas }}$ if $L \gg 2 r_{t}$ [22]. Bearing in mind uncertainties in the $L$ value in our case $(5 \lesssim L \lesssim 25 \mathrm{~mm})$, an arbitrary estimate of $S=\sqrt{S_{0} S_{\infty}}$ was

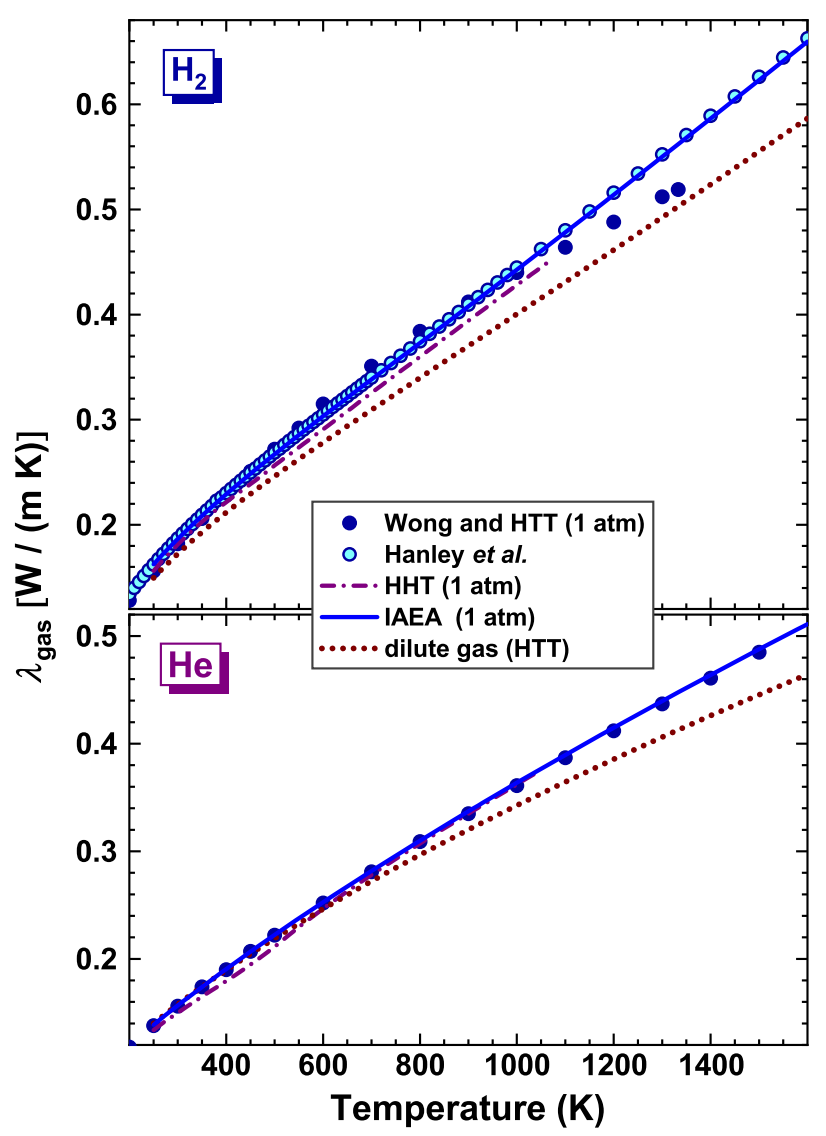

FIG. 6. Temperature dependencies of thermal conductivities for dilute $\mathrm{H}_{2}$ and $\mathrm{He}$ gases (dotted lines) obtained using Eqs. (9) and (10) [23] are shown (upper and bottom panels, respectively). The tabulated data at the atmospheric pressure [22,23,51] (circles) and those obtained with the elaborated approximations 49,52]] (lines) denoted as (HHT) and (IAEA), respectively, are also shown for comparison. 
assumed. With this assumption, the heating power removed due to gas thermal conductivity can be written as

$$
Q_{\mathrm{gas}}^{\mathrm{cnd}}=4 \sqrt{2} r_{t} \lambda_{\mathrm{gas}}\left(T_{s}-T_{0}\right) .
$$

Temperature dependencies of $Q_{\text {gas }}^{\text {cnd }}$ thus calculated are shown in Fig. 5 for $\mathrm{H}_{2}$ and $\mathrm{He}$ gases.

Heating power produced by a HI beam inside the target and backing (in W) is the product of beam intensity $I_{b}$ (in $\mathrm{p} \mu \mathrm{A}$ ) passed through the target and backing and absorbed beam energy $\Delta E$ (in $\mathrm{MeV}$ ). In a steady-state condition, this power is removed due to the summarized action of (i) thermal conductivity of the target and target backing according to Eq. (2), (ii) radiation emitted from the surfaces of the target and backing according to Eq. (3), (iii) the energy carried away by gas molecules according to Eqs. (4) or (6), or as the result of gas thermal conductivity according to Eq. (11), and (iv) heating storage in the target and backing. The equality corresponding to the energy conservation can be written as

$$
I_{b} \Delta E=Q_{\mathrm{cnd}}+Q_{\mathrm{rad}}+Q_{\mathrm{gas}}+Q_{\text {sto }} .
$$

Note that the steady-state condition is achieved within a time $t \sim 1 \mathrm{~s}$, as shown in calculations below (see Sec. III A 1). Evidently, in these conditions, storage component $Q_{\text {sto }}(T)=$ $\mathrm{cm}(d T / d t)=0$ ( $\mathrm{m}$ is the mass of a target layer/target backing and $c$ is a heat capacity). Each term in the right part of Eq. (12) is a function of target temperature $T$ determined by the beam intensity. Examples of the target temperature estimates obtained with Eq. (12) are presented below for the ${ }^{48} \mathrm{Ca}$ beam.

\section{Target temperature}

Calculations were done for a typical case innate to experiments on the synthesis of SHN, in which the ${ }^{48} \mathrm{Ca}$ input energy of $256.3 \mathrm{MeV}$ (nearby the fusion barrier of the ${ }^{48} \mathrm{Ca}+{ }^{238} \mathrm{U}$ reaction) was used in front of the $0.71 \mathrm{mg} / \mathrm{cm}^{2}$ Ti backing and $0.5 \mathrm{mg} / \mathrm{cm}^{2} \mathrm{UO}_{2}$ target. $\Delta E$, in that case, is 13.9 MeV [53]. The uniform distribution of the beam density throughout the target area $\left(r_{t}=0.5 \mathrm{~cm}\right)$ was assumed. Heating powers removed to rarefied $\mathrm{H}_{2}$ and He gases were estimated with Eqs. (6) and (11). The results are shown in Fig. 7 and compared with the calculation assuming the radiation cooling only $[5,8,11]$. As one can see, the last approaches to the estimates with Eq. (12) at high temperatures $(T>1200 \mathrm{~K})$ using the gas cooling component according to Eq. (6), due to its reduction. Such temperatures correspond to the beam intensities $I_{b}>1 \mathrm{p} \mu \mathrm{A}$ when radiation cooling becomes a primary way of heat removal.

One has to mention that the calculations mentioned above for the time-dependent radiation cooling $[5,8,11]$ can be done within the assumption of constant values for the emissivity and specific heat capacities for $\mathrm{Ti}$ and $\mathrm{UO}_{2}$,

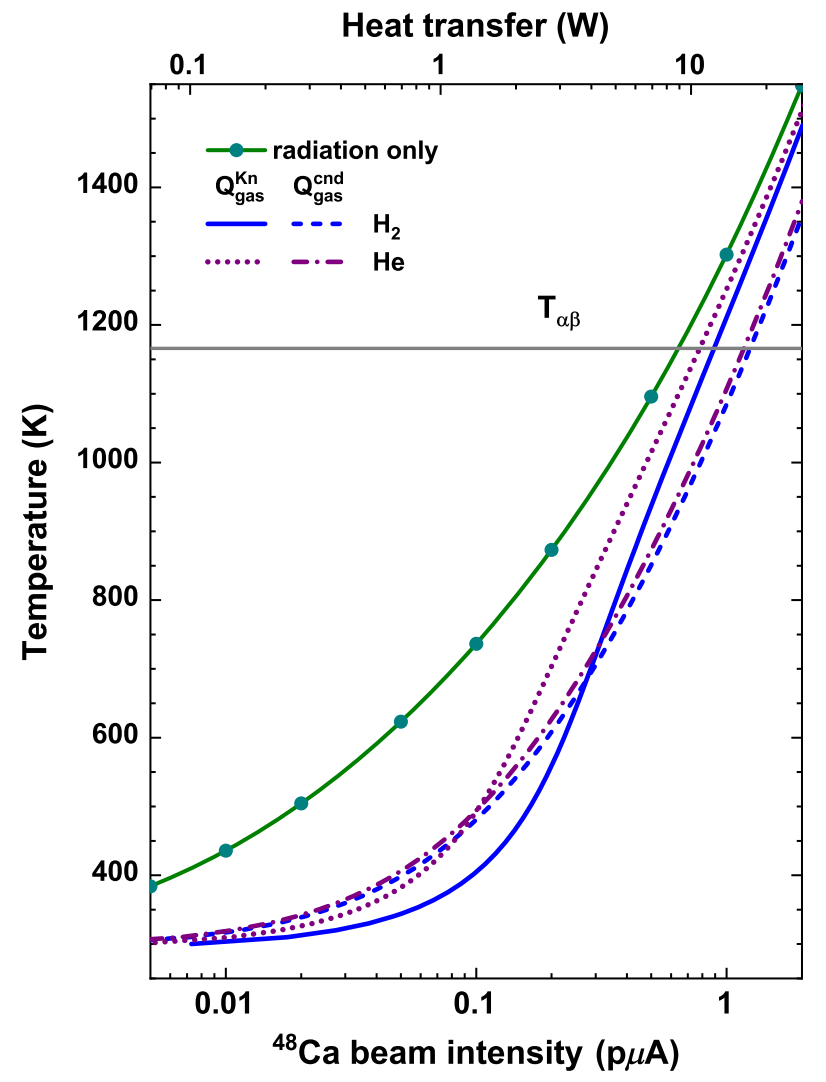

FIG. 7. Temperatures of a stationary target $\left(0.5 \mathrm{mg} / \mathrm{cm}^{2} \mathrm{UO}_{2}\right.$ on $0.71 \mathrm{mg} / \mathrm{cm}^{2}$ Ti backing) as functions of ${ }^{48} \mathrm{Ca}$ beam intensity for the uniform beam-density distribution throughout the target area $\left(r_{t}=0.5 \mathrm{~cm}\right)$ surrounded by dilute $\mathrm{H}_{2}$ or $\mathrm{He}$ gases. Estimates of heat removal to gas obtained with Eq. (6) and Eqs. (9)-(11) are designated as $Q_{\text {gas }}^{K n}$ and $Q_{\text {gas }}^{\text {cnd }}$ (respective lines). These estimates are compared to the calculations based on radiation cooling only $[5,8,11]$ (circles connected by solid lines). The temperature of the $\alpha-\beta$ transition in the Ti crystal is shown (horizontal line). The upper scale corresponds to the absorbed energy of $13.9 \mathrm{MeV}$ [53].

which is not fulfilled in a broad region of temperatures. The present calculation was done using the values at $800 \mathrm{~K}$. At beam intensity $I_{b} \lesssim 0.5 \mathrm{p} \mu \mathrm{A}$, heat removal provides acceptable target heating $(T \lesssim 1000 \mathrm{~K})$, which does not exceed the $T_{\alpha \beta}$ value corresponding to the change in the $\mathrm{Ti}$ crystal structure (see Fig. 7). This temperature can be considered as a limiting (safe) one for a target. For an actual beam distribution with the narrow Gaussian-like shape, the beam density at the beam axis (target center) is several times higher than the one for the uniform density (see Fig. 1), for which these beam-intensity dependencies were obtained.

Using the radial beam-density distribution shown in Fig. 1 and bearing in mind relatively large time of heat diffusion (see Sec. II A), the radial temperature distribution for the target can be estimated as the one composed from temperatures obtained for separate annuluses dividing the 


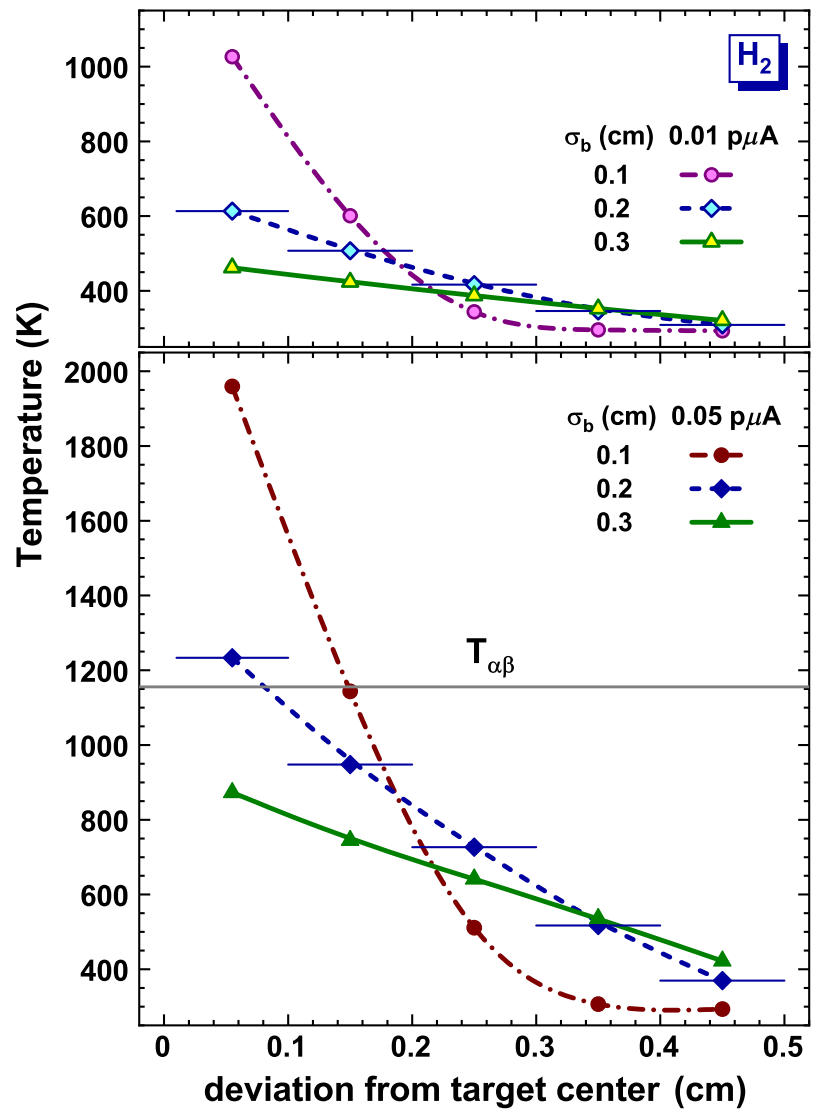

FIG. 8. Radial temperature distributions are shown (different symbols connected with respective lines) for a stationary target surrounded by rarefied $\mathrm{H}_{2}$ under irradiation of ${ }^{48} \mathrm{Ca}$ with the beam-density distribution corresponding to $\sigma_{b}=0.1-0.3 \mathrm{~cm}$ and the intensity of 0.01 and $0.05 \mathrm{p} \mu \mathrm{A}$ (upper and bottom panels, respectively). The radial beam-density distribution shown in Fig. 1 and the estimates of heat removal to gas due to its thermal conductivity were used in the estimates.

target area. This was done in the calculations of heat removal due to the thermal conductivity of the target backing and target itself, starting with $T_{0}$ at $r_{t}=0.5 \mathrm{~cm}$ and using respective modifications in Eq. (2). Heat removal to $\mathrm{H}_{2}$ gas due to its thermal conductivity was considered with the appropriate modification of Eq. (11) and using Eq. (9) for dilute gas. The estimates of heat removal from the target annuluses due to radiation were done with the respective areas entering Eq. (3).

Thus obtained distributions are shown in Fig. 8 for the same parameters of the ${ }^{48} \mathrm{Ca}$ beam as used for the uniform beam-density distribution estimates shown in Fig. 7 . Similar dependencies (with higher values for the maximal temperatures) were obtained with the use of Eq. (6), describing heat removal to $\mathrm{H}_{2}$ according to Knudsen's formula, and with Eqs. (6) and (9) applied to He gas. As one can see, narrow beam distributions $\left(\sigma_{b}=0.1-0.2 \mathrm{~cm}\right)$ at the beam intensity of $0.05 \mathrm{p} \mu \mathrm{A}$ lead to narrow temperature distributions with the maximal temperatures exceeding $T_{\alpha \beta}$. At low beam intensity $(0.01 \mathrm{p} \mu \mathrm{A})$, similar distributions may be considered safe ones for the target. Note that these estimates do not contradict the safe value of the beam intensity $(\sim 0.03 \mathrm{p} \mu \mathrm{A})$ obtained in preparation experiments with stationary $\mathrm{Er}$ and $\mathrm{Yb}$ oxide targets and ${ }^{48} \mathrm{Ca}$ beam, which are carried out during the SHN synthesis campaigns [1].

The following section will consider the effects of target rotation and beam wobbling on temperature distributions throughout the target surface.

\section{TARGET ROTATION AND BEAM WOBBLING EFFECTS ON TARGET TEMPERATURE}

In general, it seems to be evident that target rotation and beam wobbling smear a beam spot throughout a larger target area, thereby enabling a smaller beam density on average. The issue is to choose the speed of rotation and central radius $R_{c}$ of the rotating target wheel. As for the beam wobbler, the respective key parameters are the amplitude of beam deviation from the beam axis on the target surface and frequency of the wobbler.

\section{A. Effect of target rotation \\ 1. Uniform beam-density distribution}

Previous calculations of the temperature for rotating targets $[5,8,11]$ were considered in the condition of vacuum surroundings. Constant values for the emissivity and specific heat capacities of $\mathrm{Ti}$ and $\mathrm{UO}_{2}$ were assumed. These assumptions are not fulfilled in a broad region of temperatures. Calculations were performed for a rectangular beam shape and uniform beam-density distribution throughout a beam spot (target) area. Examples of such calculations as a function of time are shown in Fig. 9 that demonstrates variations in the target temperature with the beam intensity, angular velocity, and central radius of the rotating target wheel.

These calculations were performed with the same parameters of the ${ }^{48} \mathrm{Ca}$ beam and target as in previous cases (Sec. II C). The presence of a circular diaphragm with the $r_{d}=0.5 \mathrm{~cm}$ radius in front of the rotating target determined the beam density and pulse duration in the calculations. The temperatures thus obtained correspond to any point of central-circle radius $R_{c}$ or a target annulus for the circular or square diaphragm of a respective size. The parameters of the rotating targets considered in the temperature calculations are listed in Table II.

As a result of such calculations, the mean temperature is determined by the mean beam intensity depending on the ratio $t_{\mathrm{on}} / t_{\mathrm{rev}}=r_{d} /\left(\pi R_{c}\right)$ (see the upper panel in Fig. 9) and does not depend on the rotation velocity (see the bottom panel in Fig. 9). At the same time, the differences between maximal and minimal temperatures achieved for a full revolution depend on the velocity of rotation (see bottom panel in Fig. 9), in addition to the beam intensity. Figure 10 demonstrates some generalization of these 


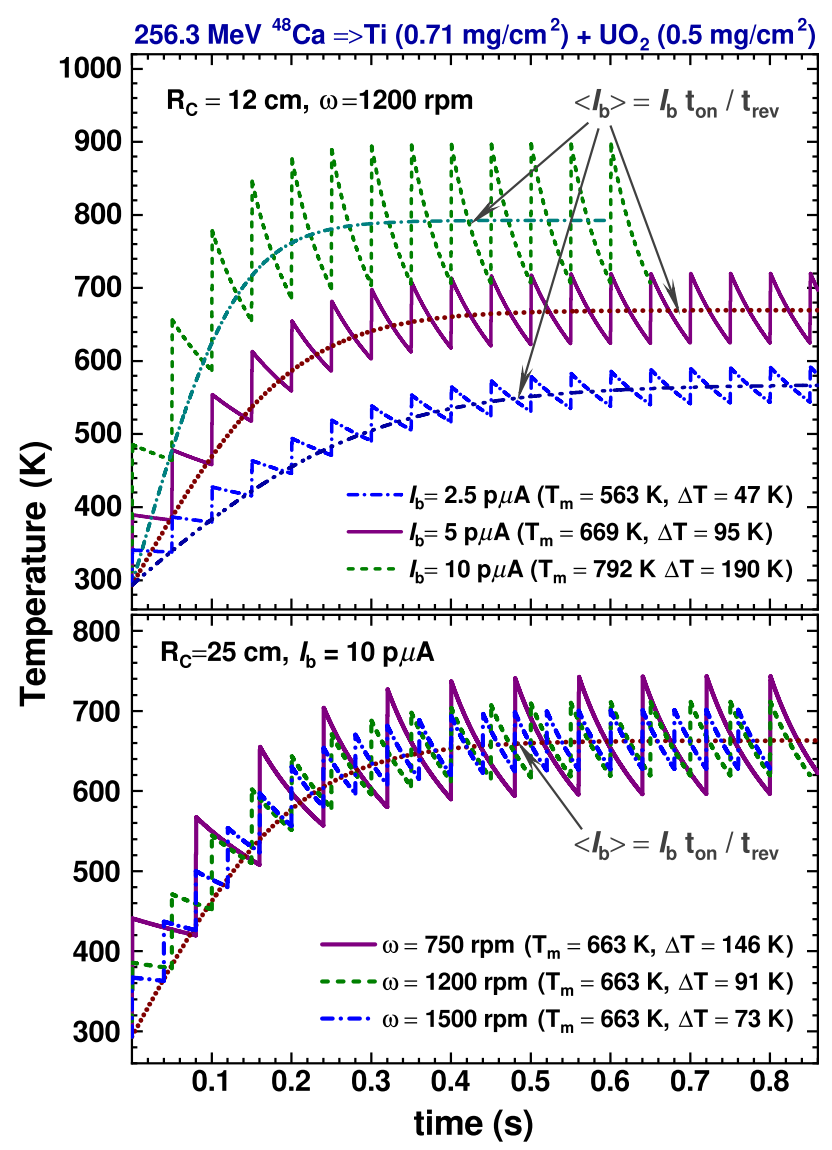

FIG. 9. Time-dependent temperature variations for a rectangular beam shape and uniform beam-density distribution are shown (different lines). These are the results of calculations (similar to Refs. $[5,8,11]$ ) for rotating targets with different central radii $R_{c}$, angular velocities $\omega$, and beam intensities $I_{b}$.

dependencies for rotation target wheels with $R_{c}=6,12$, and $25 \mathrm{~cm}$. As one can see, wheels with the largest $R_{c}$, which rotate at the highest velocity, are preferable from the point of view of their capability to withstand thermal loads corresponding to maximal $\mathrm{HI}$ beam intensities. Simultaneously, poor availability of target materials in sufficient quantity regarding heavy actinides, along with the problems of radiation protection connected with their high radioactivity, restrict the employment of such target wheels in experiments.

\section{Normal-like beam-density distribution}

With simple calculations/simulations, one can show that the beam pulse resulting from rotation corresponds to the

TABLE II. Parameters of the rotating targets.

\begin{tabular}{lccll}
\hline \hline$R_{c}(\mathrm{~cm})$ & \multicolumn{3}{c}{$\omega(\mathrm{rpm})$} & \multicolumn{1}{c}{ Note } \\
\hline 6 & 1680 & & & Used earlier [1] \\
12 & 750 & 1200 & 1500 & In use with ${ }^{242} \mathrm{Pu}$ and ${ }^{243} \mathrm{Am}$ \\
25 & 750 & 1200 & 1500 & In project \\
\hline \hline
\end{tabular}

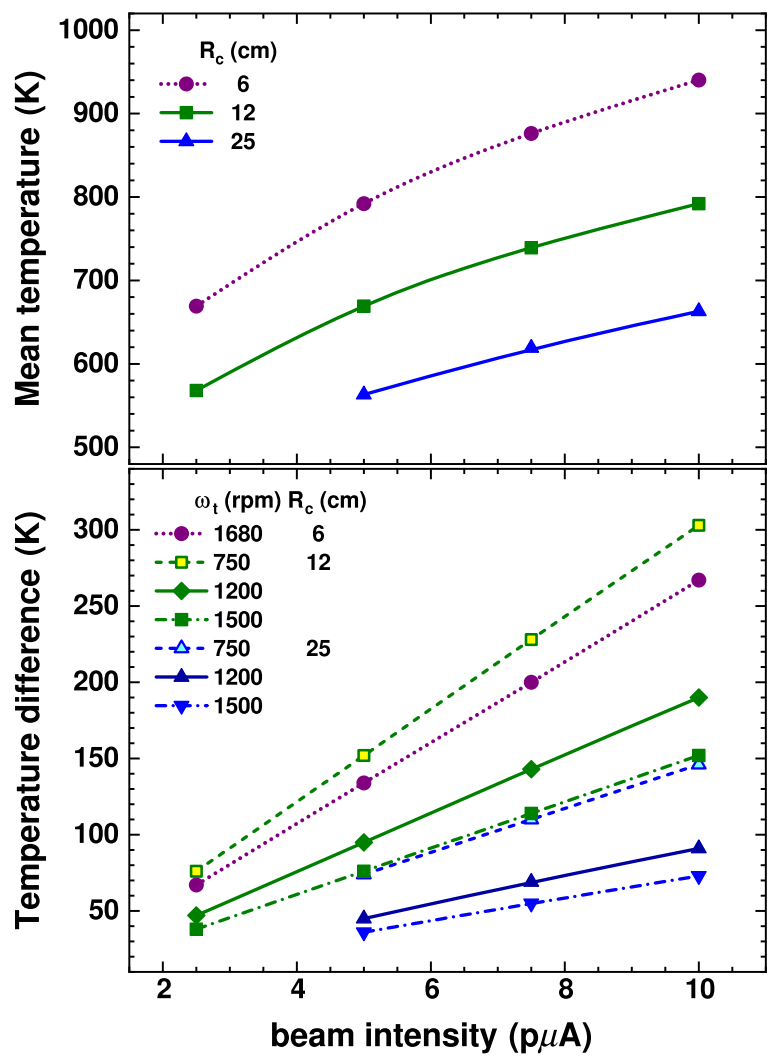

FIG. 10. Mean temperatures and differences between maximal and minimal temperatures as a function of ${ }^{48} \mathrm{Ca}$ beam intensity for rotating target wheels with different central radii $R_{c}$ and angular velocities $\omega$.

uniform density distribution in the rotation $(x)$ direction and keeps its form in the perpendicular $(y)$ direction for a realistic normal-like beam-density distribution. In Fig. 11, $y$ distributions are presented for $\sigma_{b}=\sigma_{y}=0.1-0.3 \mathrm{~cm}$ compared with the uniform one cut by the $1 \mathrm{~cm}$ annulus width of the rotating target. Note that beam losses $\varepsilon_{\text {loss }}$, in that case, are lower than those for the respective stationary target (see Fig. 1).

Further estimates of temperature distributions are considered below, similarly to the one for a stationary target, i.e., in steady-state conditions with beam distribution widths $\sigma_{b}=\sigma_{y}=0.1-0.3 \mathrm{~cm}$, but with the beam intensity corresponding to mean value $\left\langle I_{b}\right\rangle=I_{b} t_{\text {on }} / t_{\text {rev }}$.

\section{B. Effect of beam wobbling}

The effect of beam wobbling is below considered using the example of a device installed in the beam line of the FLNR high-current cyclotron DC280. The wobbler is installed at a certain distance from the rotating target and provides sinusoidal beam oscillations of the respective amplitude and frequency on the target surface. In experiments, the amplitude is adjusted to provide beam losses at the level not exceeding (10-15)\% of the beam intensity measured against the switched-off wobbler. Since the 


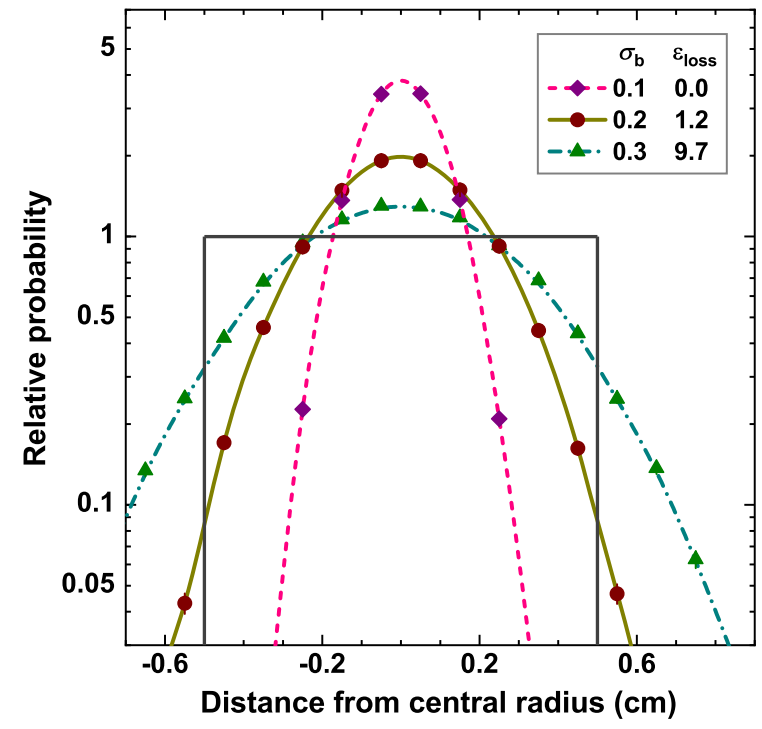

FIG. 11. Normal beam-density distributions throughout the target surface in the direction perpendicular to the rotating direction, for $\sigma_{b}=\sigma_{y}=0.1-0.3 \mathrm{~cm}$, resulting from simulations (symbols connected by lines). Beam losses $\varepsilon_{\text {loss }}$ (in \%) corresponding to the beam cut by the $1 \mathrm{~cm}$ annulus width of the rotating target are indicated for the respective values of $\sigma_{b}$. The beam distributions are compared to the uniform distribution assumed in the time-dependent temperature calculations considered in Sec. III A 1 (straight solid lines).

operation of the wobbler is not synchronized with target rotation, the spreading effect produced by the wobbler within a beam pulse depends on the velocity of rotation and oscillation frequency. Device performance is illustrated with calculations of the $Y_{p}(t)$ position for the "point" beam $\left(\sigma_{b}=0\right)$ on the target surface at the amplitude of beam deflection $A_{w}$ provided by the wobbler:

$$
Y_{p}(t)=A_{w} \sin \left\{2 \pi f_{w}\left[t+N_{\text {rev }}\left(t_{\text {rev }}-t_{\text {on }}\right)\right]\right\},
$$

where $f_{w}$ is the deflection frequency and $N_{\text {rev }}$ is the number of full revolutions of the target wheel.

The examples of calculations are shown in Fig. 12. They were done for $f=500 \mathrm{~Hz}, 20$ complete revolutions of the target wheel, the beam pulse determined by the $1 \mathrm{~cm}$ arc of the circle radius $R_{c}=12 \mathrm{~cm}$, and the angular velocities of 750 and $1500 \mathrm{rpm}$. As we can see, within 17 and 15 revolutions at the velocity of 750 and $1500 \mathrm{rpm}$, respectively, the beam trajectories cover the target annulus area in a regular way. With the increasing number of revolutions, subsequent trajectories are approached to those from previous revolutions. Similar calculations for the target with $R_{c}=6 \mathrm{~cm}$ at $\omega=1500 \mathrm{rpm}$ show, as one could expect, the same picture as obtained with $R_{c}=12 \mathrm{~cm}$ at $\omega=750 \mathrm{rpm}$ (the left panel in Fig. 12). For the same target at $\omega=750 \mathrm{rpm}$, two times higher trajectory density is obtained, comparing with the picture observed at $\omega=1500 \mathrm{rpm}$. In the case of a large target wheel with

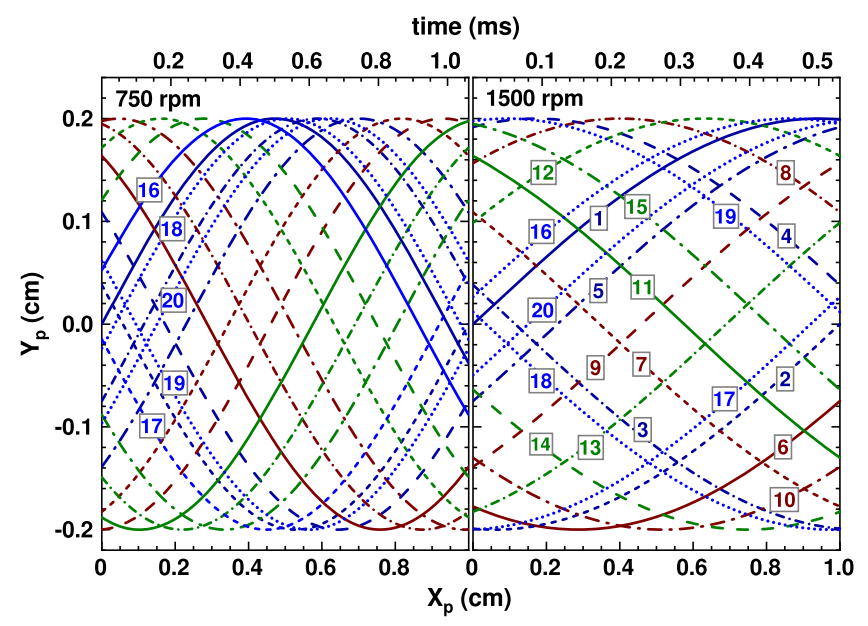

FIG. 12. Beam trajectories within the beam pulse corresponding to the 1-cm arc of the circle radius $R_{c}=12 \mathrm{~cm}$, as calculated for the "point" beam $\left(\sigma_{b}=0\right)$ and the $0.2 \mathrm{~cm}$ amplitude of beam deflection produced by the wobbler on the target surface. The calculations were done for the target velocities of 750 and $1500 \mathrm{rpm}$, and 20 complete target wheel revolutions (the number of revolutions is indicated in squares). Thus obtained trajectories are shown by five different lines (from solid to dash-dotted ones) of the same color. Trajectories obtained after 17 and 15 revolutions (shown by dotted lines) at the velocity of 750 and $1500 \mathrm{rpm}$, respectively, are approaching those obtained for the previous target wheel revolutions.

$R_{c}=25 \mathrm{~cm}, 55$ complete revolutions have to be done in order to achieve a uniform trajectory density throughout the same area.

In MC simulations with finite beam width, these numbers of revolutions would be sufficient to get an idea of the beamdensity distribution providing pulse heating during the target rotation and beam wobbling. Such simulations were performed for the target with $R_{c}=12 \mathrm{~cm}$ at the velocities of 750 and $1500 \mathrm{rpm}$, beam widths $\sigma_{b}=0.1-0.4 \mathrm{~cm}$, and wobbling amplitudes $A_{w}=0.1-0.5 \mathrm{~cm}$. In Fig. 13, the results of such simulations for $\sigma_{b}=0.2 \mathrm{~cm}, A_{w}=0.4 \mathrm{~cm}$, and $\omega=1500 \mathrm{rpm}$ are shown for example.

In Fig. 14, beam-density distributions integrated over $X_{p}$ within the $1 \mathrm{~cm}$ arc length for the target with $R_{c}=12 \mathrm{~cm}$ are shown for different $A_{w}, \sigma_{b}=0.1$ and $0.2 \mathrm{~cm}$. These distributions, along with $\mathrm{MC}$ simulations, similar to those shown in Fig. 13, were used for the estimates of beam losses caused by diaphragms installed in front of the target for the different $A_{w}, \omega$, and $\sigma_{b}$ values.

In Fig. 15, the values of beam losses resulting from MC simulations are shown for different beam widths, wobbling amplitudes, and diaphragms installed in front of the rotating target with $R_{c}=12 \mathrm{~cm}$, for $\omega=750$ and $1500 \mathrm{rpm}$. As one can see, the $A_{w}$ dependencies obtained for the same beam widths and different velocities are close to each other, despite the different beam trajectories drawn by the wobbler on the target surface (see Fig. 12). 


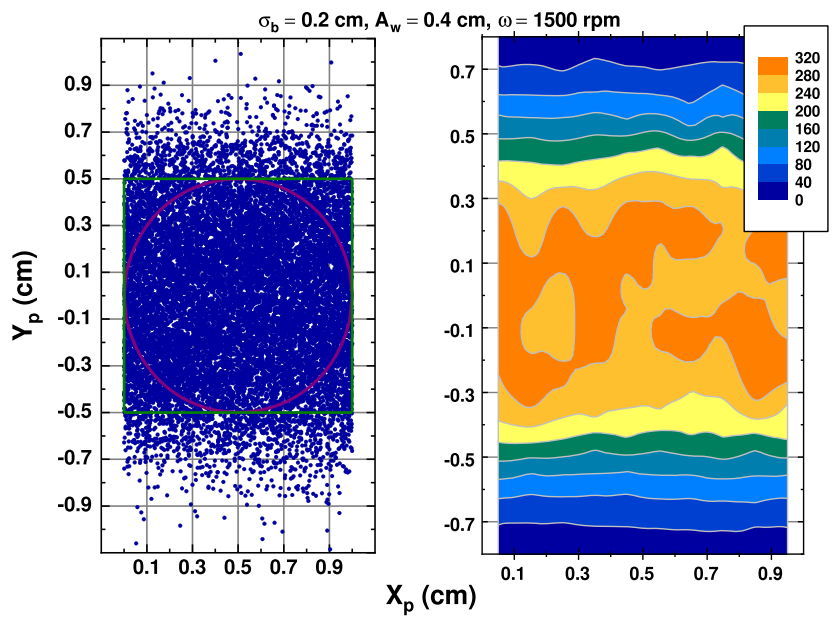

FIG. 13. The result of $3 \times 10^{4} \mathrm{MC}$ simulations for beam width $\sigma_{b}=0.2 \mathrm{~cm}$ and wobbling amplitude on the target surface $A_{w}=0.4 \mathrm{~cm}$ is shown for the rotating target with central radius $R_{c}=12 \mathrm{~cm}$ and rotating velocity $\omega=1500 \mathrm{rpm}$ (left panel). The same distribution reduced to the number of events collected by the $0.1 \times 0.1 \mathrm{~cm}$ cell and subsequently smoothed is shown in the right panel as a contour map. Frames corresponding to the circular and square diaphragm of respective sizes are shown in the left panel.

Within the same simulations, quantitative estimates of the nonuniformity of the beam density over the target surface were obtained in the case of a square diaphragm of $1 \times 1 \mathrm{~cm}$, which cut the same square on the target annulus. This characteristic was obtained as the ratio of the standard deviation to the average value for the number of events collected in $0.1 \times 0.1 \mathrm{~cm}$ cell. The results are shown in Fig. 16 for the rotating target with $R_{c}=12 \mathrm{~cm}$, at $\omega=750$

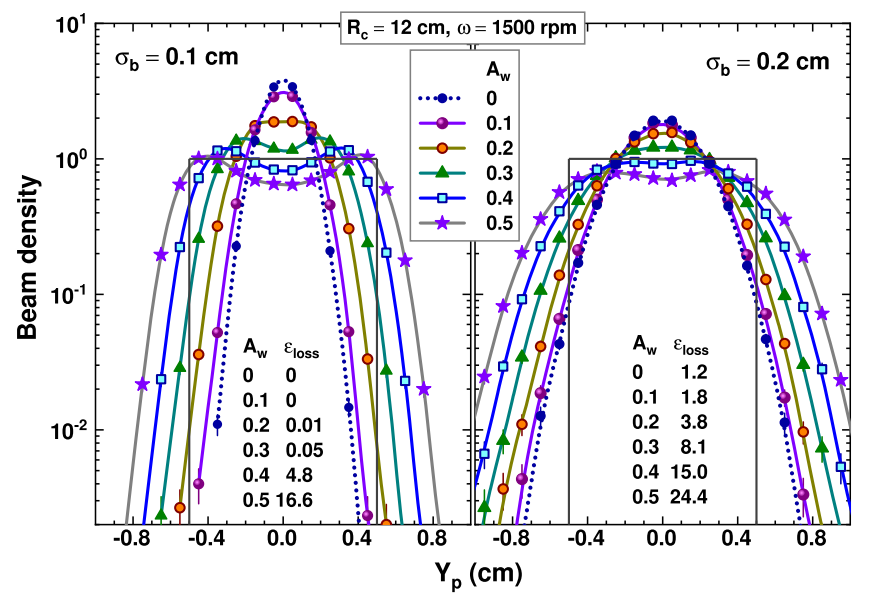

FIG. 14. Beam-density distributions integrated over $X_{p}$ within the 1-cm arc length for the target with $R_{c}=12 \mathrm{~cm}$ are shown for the indicated velocity $\omega$, wobbling amplitude $A_{w}$ (in $\mathrm{cm}$ ), and beam widths $\sigma_{b}$ (symbols connected by lines). These distributions are compared to the uniform distribution with the unit beamdensity probability. Straight lines at $Y_{p}= \pm 0.5 \mathrm{~cm}$ correspond to the annulus width of the rotating target, which determines beam losses $\varepsilon_{\text {loss }}$ indicated in $\%$.

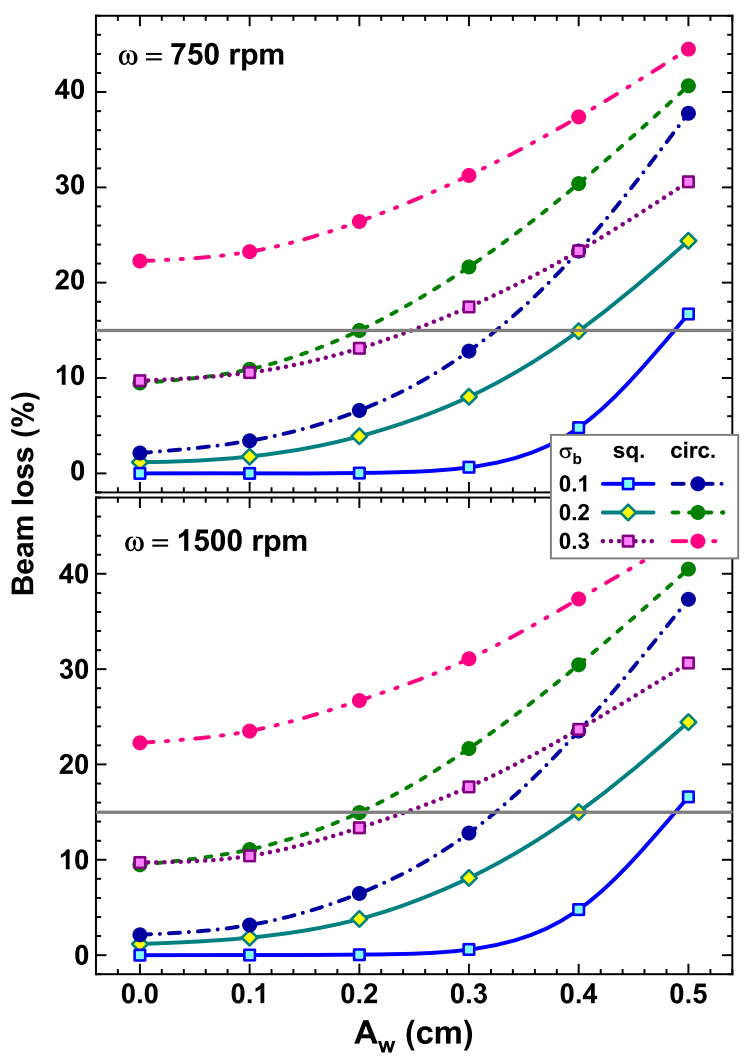

FIG. 15. Beam losses derived with MC simulations for beam widths $\sigma_{b}=0.1-0.3 \mathrm{~cm}$, and wobbling amplitudes $A_{w}=0-0.5 \mathrm{~cm}$ as applied to the rotating target with $R_{c}=12 \mathrm{~cm}$, for the velocities of 750 and $1500 \mathrm{rpm}$ (upper and bottom panels, respectively), and different diaphragms installed in front of the target (symbols connected by lines). Data correspond to the square and circular diaphragm of the size of $1 \times 1 \mathrm{~cm}$ and $1 \mathrm{~cm}$ in diameter designated as (sq.) and (circ.), respectively. Straight solid lines corresponding to the $15 \%$ beam loss are shown in both panels for orientation.

and $1500 \mathrm{rpm}$. As one can see, the values of the nonuniformity thus obtained for $\sigma_{b}=0.2-0.3 \mathrm{~cm}$ approach to zero with the increase in the wobbling amplitude.

Figures 16 and 15 joint viewing shows that the least nonuniformities of the beam density on the target surface and minor beam losses are achieved at $A_{w}=0.3-0.4 \mathrm{~cm}$ for beam widths $\sigma_{b}=0.1-0.2 \mathrm{~cm}$. That is in qualitative accordance with the respective $Y_{p}$ distributions, which are close to the uniform beam-density distribution, as shown in Fig. 14. Further estimates of the rotating target temperature could be done similarly to those performed for the stationary target (see results in Fig. 7), i.e., in steady-state conditions, but for the beam intensity corresponding to the mean value $\left\langle I_{b}\right\rangle=I_{b} t_{\mathrm{on}} / t_{\text {rev }}$.

\section{Target temperature for rotating target and beam wobbling}

As in previous cases, estimates of the temperature for rotating targets were done for the ${ }^{48} \mathrm{Ca}$ input energy of 


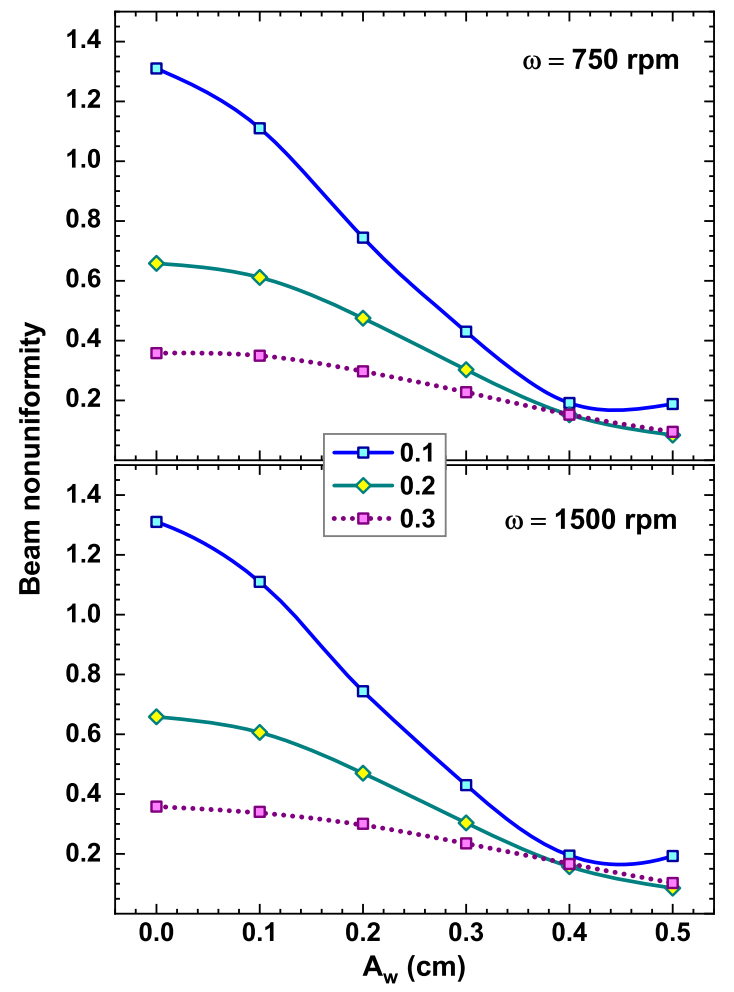

FIG. 16. The same as in Fig. 15, but for the beam nonuniformities resulting from the MC simulations for the square diaphragm of $1 \times 1 \mathrm{~cm}$. See details in the text.

256.3 MeV in front of the $0.71 \mathrm{mg} / \mathrm{cm}^{2} \mathrm{Ti}$ target backing and $0.5 \mathrm{mg} / \mathrm{cm}^{2} \mathrm{UO}_{2}$ target. The uniform beam-density distribution throughout a target area was assumed, which could be approximately reached for $A_{w}=0.4 \mathrm{~cm}$, and $\sigma_{b}=0.1$ and $0.2 \mathrm{~cm}$ as shown in Figs. 12-14. In that cases, the respective mean-weighted densities of $0.969 \pm$ 0.024 and $0.859 \pm 0.016$ are slightly less than the unit corresponding to the uniform distribution. These reductions were neglected in further considerations. As well as in the case of rotating targets under radiative cooling only (Sec. III A 1), mean temperatures thus estimated correspond to the steady-state regime which is established throughout the rotating target area.

Some additional details were further considered. Thus, one should take into account that the square diaphragm of $1 \times 1 \mathrm{~cm}$ provides minor beam losses for the rotating target. As a consequence, in Eqs. (3) and (6), for the heating power removed by radiation and dilute gas, respectively, area $F$ should correspond to the square area.

For the estimates of heating powers removed due to the thermal conductivity of the target material and target backing as well as those of dilute gas, which were expressed with Eqs. (2) and (11), respectively, other formulas, corresponding to the "rectangular geometry" of heated elements [22] were applied in considering these processes:

$$
\begin{gathered}
Q_{\mathrm{cnd}}=\frac{2[5.7+b /(2 a)]}{\ln \left[3.5 L /\left(b^{1 / 4} a^{3 / 4}\right)\right]} \lambda D\left(T_{s}-T_{0}\right), \\
Q_{\mathrm{gas}}^{\mathrm{cn} 1}=\frac{2 \pi b \lambda_{\mathrm{gas}}}{\ln (4 b / a)}\left(T_{s}-T_{0}\right), \\
Q_{\mathrm{gas}}^{\mathrm{cn} 2}=\frac{\pi b \lambda_{\mathrm{gas}}}{\ln (4 b / a)}\left(T_{s}-T_{0}\right) .
\end{gathered}
$$

Eq. (14) was used instead of Eq. (2) for the estimates of heating powers removed due to the thermal conductivity of the Ti target backing and $\mathrm{UO}_{2}$ target itself. In Eqs. (14)-(16), $a$ and $b$ are the lengths of rectangle sides corresponding to $a=b=1 \mathrm{~cm}$ in our case; $L$ is the distance from central radius $R_{c}$ (target surfaces) to a cold (surrounding) target frame. The relations of $L=a / 2+0.2$ and $L=a / 2$ were assumed for the estimates of heating powers removed due to the thermal conductivity of the Ti backing and $\mathrm{UO}_{2}$ target, respectively. The heating power removed due to gas thermal conductivity was estimated as $Q_{\mathrm{gas}}^{\mathrm{cnd}}=\sqrt{Q_{\mathrm{gas}}^{\mathrm{cn} 1} Q_{\mathrm{gas}}^{\mathrm{cn} 2}}$ that implies yielding Eq. (15) if $L>2 a$ and Eq. (16) if $L=0$ [22]. It is similar to considering the heating power removed by the thermal conductivity of gas surrounding a stationary target with the use of Eq. (11), taking into account uncertainties in the $L$ value.

As well as in consideration of heat transfer in a steadystate condition for the stationary target using Eq. (12), the power released inside the rotating target and target backing is removed from them by the combined action of (i) thermal conductivity of the target and target backing, (ii) radiation emitted from the surfaces of the target and backing, and (iii) gas, due to the energy carried away by gas molecules or gas thermal conductivity. In Fig. 17, temperatures of the rotating targets with different central radii $R_{c}$ are shown in the same way as was done for the stationary target of a $0.5 \mathrm{~cm}$ radius (see Fig. 7). As in the previous case, estimates of heating power removed to rarefied $\mathrm{H}_{2}$ and He gases surrounding the rotating target were performed with Knudsen's formula using Eq. (6), with Eqs. (15) and (16) as described above when heat removal due to the thermal conductivity process was considered.

Comparing Figs. 7 and 17, the benefits of rotating targets over stationary ones are clearly seen from the point of view of reducing a thermal load. Nevertheless, there is an issue requiring attention when these estimates are compared. The heat transfer estimates due to the Ti backing and $\mathrm{UO}_{2}$ target's thermal conductivity were obtained, assuming that their cooling edges have the room temperature $T_{0}=293.15 \mathrm{~K}$ for the stationary target and rotating ones. This condition seems to be better fulfilled for the stationary target, since it could be installed on a massive copper target holder cooled by cold water. Simultaneously, separate target cells, consisting of a rotating target annulus, 


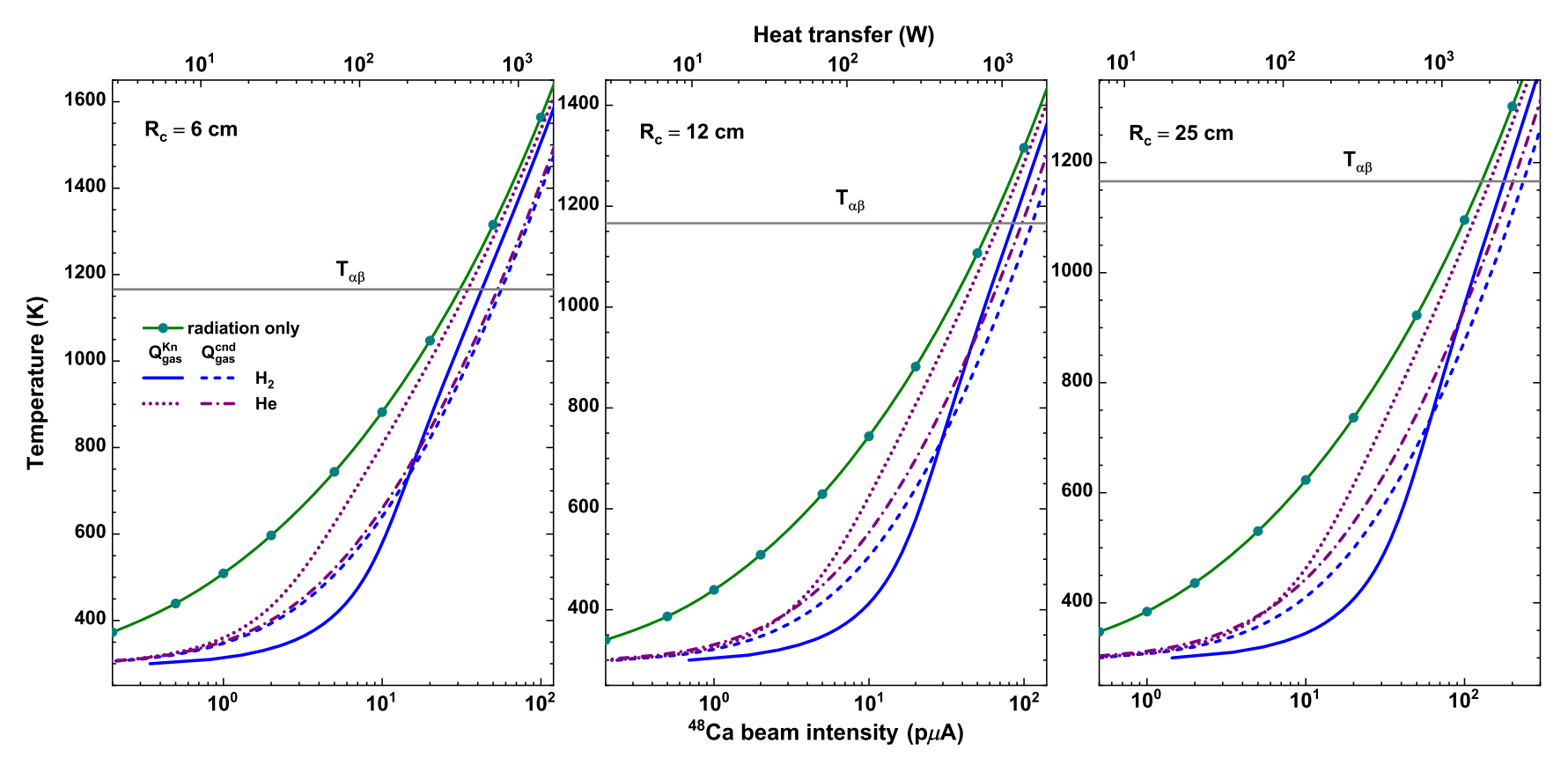

FIG. 17. The same as in Fig. 7, but for the rotating targets with central radii $R_{c}=6,12$, and $25 \mathrm{~cm}$.

suffer from a less effective heat removal to their frames since the target wheel is made of light material (usually it is a duralumin disk of $2 \mathrm{~mm}$ of thickness), which rotates without any forced cooling. Consequently, the temperatures at the edges of a target annulus may not differ so much from the mean temperature of the target. Below this circumstance is considered in more detail.

\section{Effect of thermal conductivity on target temperature}

Estimates of the total heating power removed from rotating targets using Eq. (12) show that the contribution of the target and backing thermal conductivity into the summarized value of heat removal is not significant. For example, increasing target temperature from 400 to $1000 \mathrm{~K}$ leads to decreasing the relative contributions of the thermal conductivity process from $3.1 \%$ to $1.1 \%$, irrespective of the target radius. Bearing in mind minor heat removal from the edges of the rotating target annulus, as was above mentioned, one may assume that the temperatures of both circumferences of smaller and larger radii $R_{1}$ and $R_{2}$ are the same and equal to the mean temperature determined by the total heating power released into and removed from the target. With this assumption, the temperature distribution over the target annulus width could be obtained by the solution of the differential equation:

$$
\frac{d^{2} T}{d r^{2}}+\frac{1}{r} \frac{d T}{d r}=-\frac{Q_{\mathrm{cnd}}}{\lambda V_{\mathrm{tar}}}
$$

where $Q_{\text {cnd }}$ is the heating power that could be only removed due to thermal conductivity, i.e., applying Eq. (14) and
$V_{\text {tar }}$ is the target volume. This equation is analytically solved using boundary conditions determined as $T\left(R_{1}\right)=$ $T\left(R_{2}\right)=T_{m}$, where $T_{m}$ is the mean temperature corresponding to the total heating power removed from the target, as shown in Fig. 17. Such boundary conditions simplify the calculations within this approach. However, unfortunately, more reliable conditions cannot be established because of difficulties in determining the (contact) conductivity at the edges of the target backing.

In Fig. 18, temperature distributions thus obtained are shown for the rotating targets with $R_{c}=6,12$, and $25 \mathrm{~cm}$, and the beam intensity varied within $0.5-20 \mathrm{p} \mu$ A. Targets are surrounded by rarefied $\mathrm{H}_{2}$. Thermal conductivity coefficients corresponding to respective $T_{m}$ values were used in the estimates. The largest temperature excess over the mean value at $r=R_{c}$ is not as significant as one could expect. It is $42 \mathrm{~K}$ for the relatively small target with $R_{c}=6 \mathrm{~cm}$ and the beam intensity of $20 \mathrm{p} \mu \mathrm{A}$.

\section{SUMMARY}

The effects of target rotation and beam wobbling on the temperature load produced by an intense HI beam were quantitatively considered. The study is motivated by extending investigations on the synthesis and properties of SHN. These nuclei are produced in the complete-fusion reactions induced by $\mathrm{HI}$ projectiles on actinide target nuclei with extremely low cross sections caused by the reaction mechanism. More intense $\mathrm{HI}$ beams of ${ }^{48} \mathrm{Ca}$ and heavier projectiles are needed to obtain a sufficient number of SHN decay events to establish their properties. Increasing the intensity of HI beams increases the heating power released 

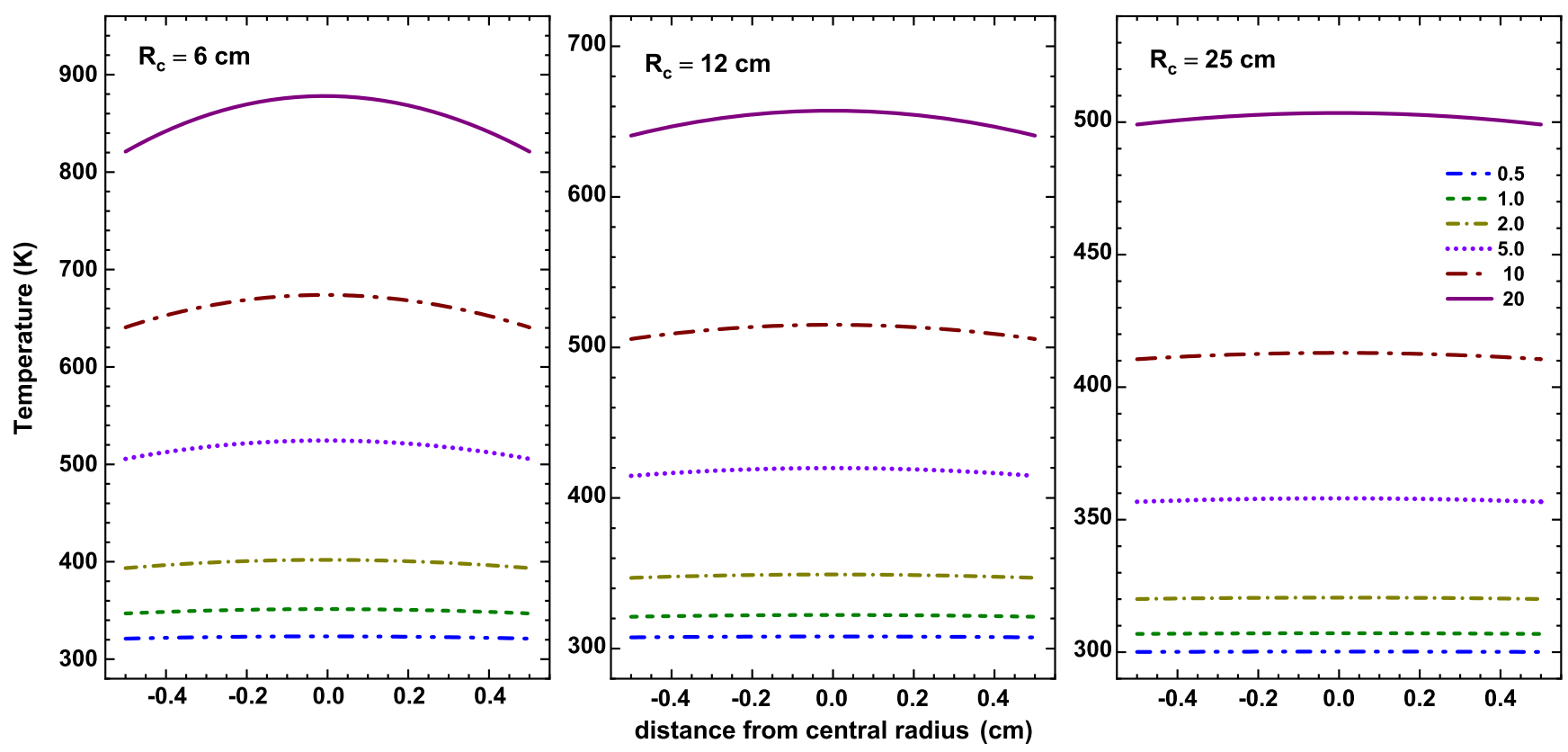

FIG. 18. Temperature distribution across the width annulus for the rotating targets with central radius $R_{c}=6,12$, and $25 \mathrm{~cm}$ (panels from left to right) at the beam intensity varied within $0.5-20 \mathrm{p} \mu \mathrm{A}$ (different lines). The pedestal temperatures for each distribution correspond to the mean values shown in Fig. 17 for rarefied $\mathrm{H}_{2}$ (dashed lines in the figure panels), at which gas thermal conductivity values were used in the estimates of respective heating power removal.

inside the targets and target backings used in experiments. A permanent thermal load may restrict the durability of targets and, as a consequence, the duration of experiments.

The way to increase the durability of targets is to use rotating targets with large irradiated areas and pulse temperature loads. The specific heating load is essentially decreased in such targets due to spreading a high power released inside the relatively small target and target backing thicknesses. At present, the rotating targets of actinideoxide material deposited on the Ti backing are used in experiments on the synthesis of SHN in laboratories worldwide. Unfortunately, an increase in sizes (areas) of a rotating target is limited by an available amount of heavyactinide target materials and radiation safety considerations. One more problem that cannot be entirely solved with target rotation is a narrow beam distribution which produces enhanced beam density in the vicinity of the circumference of a central radius of the target annulus. The way to solve this problem is using beam wobbling that spreads a beam in the direction perpendicular to the direction of target rotation.

In the conditions of gas-filled recoil separators used in experiments on the synthesis of SHN, a rotating target operates in the atmosphere of rarefied $\mathrm{H}_{2}$ or He gases. A heating power released inside the target and target backing can be removed due to the processes of (i) the target and target backing thermal conductivity transferring heat to the target frames, (ii) radiation emitted from the surfaces of the target and target backing, and (iii) heat transfer to gas surrounding the target.
Each of the heat removal processes was initially considered for a stationary target and a uniform beam-density distribution throughout the target area. In the framework of this consideration, temperature dependencies for the thermal conductivity coefficient and hemispherical emissivity of Ti have been obtained, which are based on available experimental data. The treatment of heat removal to gas has been led to empirical dependencies for the accommodation coefficients of $\mathrm{H}_{2}$ and $\mathrm{He}$ molecules on heated Ti and $\mathrm{UO}_{2}$ surfaces, which are also based on available experimental data. Thus obtained, the temperature dependencies were used for the estimates of heat transfer to gas according to the models of heat transfer to the kinetic energy of gas molecules. The estimates based on the thermal conductivity of dilute gas and gas at atmospheric pressure were also performed when considering the heat transfer to gas.

For example, this approach was applied to the stationary target of a $0.5 \mathrm{~cm}$ radius and ${ }^{48} \mathrm{Ca}$ beam with the uniform beam-density distribution and input energy of $256.3 \mathrm{MeV}$. The total energy absorbed by the Ti backing and $\mathrm{UO}_{2}$ target itself is $13.9 \mathrm{MeV}$ in that case. The results have shown that heating powers removed from the target in the frameworks of different approaches are about the same (differ from each other within a factor of $\sim 2$ ) at temperatures $T \lesssim 800 \mathrm{~K}$. Above this temperature, the estimates corresponding to the heat transfer to the kinetic energy of gas molecules show some decrease in heating power removed due to respective decrease in the accommodation coefficients. Such behavior contrasts with the one corresponding to the gas thermal conductivity, which gradually increases 
with the temperature. At $T \gtrsim 1000 \mathrm{~K}$, this heating power becomes comparable with the one corresponding to the radiation cooling, the dominant way of target cooling at high temperatures. Within these estimates, ${ }^{48} \mathrm{Ca}$ beam intensities up to $0.5 \mathrm{p} \mu \mathrm{A}$ could be considered safe for a stationary target irradiated by the beam with the uniform beam density.

For an actual beam distribution with a narrow Gaussianlike shape, and the beam density exceeding by several times the uniform density at the beam axis (target center), the temperature distribution along the target radius was estimated for separate annuluses dividing the stationary target area. Respective modifications of formulas for heating powers removed due to the thermal conductivity of dilute gas and radiation emission were used in the estimates. Narrow beam-density distributions $\left(\sigma_{b}=0.1-0.2 \mathrm{~cm}\right)$, with the same parameters of ${ }^{48} \mathrm{Ca}$ beam as used for the estimates using the uniform beam density, lead to narrow temperature distributions with the maximal temperatures exceeding the temperature of crystal transition $T_{\alpha \beta}$ in Ti at the beam intensity of $0.05 \mathrm{p} \mu \mathrm{A}$. Similar distributions may be considered safe for the target at a lower beam intensity $(0.01 \mathrm{p} \mu \mathrm{A})$. These estimates do not contradict the safe value for the ${ }^{48} \mathrm{Ca}$ beam intensity $(\sim 0.03 \mathrm{p} \mu \mathrm{A})$ established in preparation experiments with stationary $\mathrm{Er}$ and $\mathrm{Yb}$ oxide targets [1].

Target rotation leads to the pulse heating of the same area on a target annulus with a respective rise and fall with a time. Such behavior depends on the size of a beam spot in the direction of rotation or a diaphragm installed in front of the target, angular velocity, and central radius $R_{c}$. For the uniform beam density and assuming that radiation is the only way of heat removal from the target, the temperature as a function of time could be presented explicitly as showing a sawtooth dependence. For the fixed $R_{c}$ and beam intensity $I_{b}$, the amplitude of temperature variations (a difference between maximal and minimal temperatures) in the steady-state condition decreases with increasing the rotation velocity. The amplitude of pulsed temperature increases with increasing $I_{b}$ and decreasing $R_{c}$. Simultaneously, the mean temperature corresponding to the steady-state condition is independent of the rotation velocity for the fixed $R_{c}$ and mean beam intensity. The last is determined by the relationship $\left\langle I_{b}\right\rangle=I_{b} t_{\mathrm{on}} / t_{\mathrm{rev}}=$ $I_{b} d /\left(2 \pi R_{c}\right)$, where $t_{\text {on }}$ and $t_{\text {rev }}$ are the transit time of the beam spot (diaphragm with characteristic size $d$ ) size and the time of complete target revolution, respectively.

Using beam wobbling allows one to achieve a quasiuniform beam-density distribution throughout the surface of the rotating target. That was shown with calculations and $\mathrm{MC}$ simulations for the targets with different central radii and angular velocities. From the point of view of the least beam losses and nonuniformity of the beam density, the best results were achieved for relatively narrow beam-density distributions corresponding to width $\sigma_{b}=0.1-0.2 \mathrm{~cm}$ at wobbling amplitude on the target surface $A_{w}=0.3-0.4 \mathrm{~cm}$. For the steady-state condition, the mean temperature as a function of $I_{b}$ was estimated in the same way as was done for a stationary target irradiated by the beam with the uniform beam density and mean intensity $\left\langle I_{b}\right\rangle$. As the results of these estimates, a conditionally dangerous temperature, corresponding to the $\alpha-\beta$ transition in the crystal structure of $\mathrm{Ti}$, is achieved at the ${ }^{48} \mathrm{Ca}$ beam intensities exceeding 30,70, and $130 \mathrm{p} \mu \mathrm{A}$ for the rotating targets with $R_{c}=6,12$, and $25 \mathrm{~cm}$, and $\mathrm{He}$ as surrounding gas. The upper limits for thus obtained intensities depend upon how the heat transfer to rarefied $\mathrm{H}_{2}$ or He gas is estimated.

One would think that the present approach can be applied for any combination of heavy ion projectile interacting with any target and target backing to estimate a thermal load on them. These estimates are necessary either for a stationary and rotating target in assessing their durability over prolonged experiments.

[1] Yu. Ts. Oganessian and V. K. Utyonkov, Rep. Prog. Phys. 78, 036301 (2015).

[2] K. Subotic, Yu. Ts. Oganessian, V. K. Utyonkov, Yu. V. Lobanov, F. Sh. Abdullin, A. N. Polyakov, Yu.S. Tsyganov, and O. V. Ivanov, Nucl. Instrum. Methods Phys. Res. Sect., A 481, 71 (2002).

[3] G. G. Gulbekian, S. N. Dmitriev, Yu. Ts. Oganessian, B. N. Gikal, I. V. Kalagin, S. L. Bogomolov, I. A. Ivanenko, N. Yu. Kazarinov, G. N. Ivanov, and N. F. Osipov, in Proceedings of the 21st International Conference on Cyclotrons and their Applications, Zurich, Switzerland, 2016, p. 278, http://accelconf.web.cern.ch/AccelConf/ cyclotrons $2016 /$.

[4] V. I. Zagrebaev and W. Greiner, Nucl. Phys. A944, 257 (2015).

[5] J. L. Yntema and F. Nickel, in Experimental Methods in Heavy Ion Physics, edited by K. Bethge, Lecture Note of Physics Vol. 78 (Springer, New York, 1978), p. 206.

[6] J. O. Lilijenzin, Lawrence Berkeley Laboratory Report, Technical Report No. LBL-1912, 1973.

[7] P. Cagarda, Ph.D. Thesis, Comenius University, Bratislava, 2002.

[8] R. N. Sagaidak, Phys. Part. Nucl. Lett. 14, 747 (2017).

[9] N. Yu. Kazarinov, G. G. Gulbekian, and V. I. Kazacha, Phys. Part. Nucl. Lett. 15, 319 (2018).

[10] D. Kaji, K. Morimoto, A. Yoneda, H. Hasebe, A. Yoshida, H. Haba, S. Goto, H. Kudo, and K. Morita, Nucl. Instrum. Methods Phys. Res. Sect., A 590, 198 (2008).

[11] D. Marx, F. Nickel, G. Münzenberg, K. Güttner, H. Ewald, W. Faust, S. Hofmann, H. J. Schött, and W. Thalheimer, Nucl. Instrum. Methods Phys. Res., Sect. A 163, 15 (1979).

[12] C. Stodel, F. Pellemoine, R. Hue, F. Lutton, C. Marry, and J.-F. Libin, Nucl. Instrum. Methods Phys. Res., Sect. A 613, 480 (2010). 
[13] K. Eberhardt et al., Nucl. Instrum. Methods Phys. Res., Sect. A 590, 134 (2008).

[14] E. Jäger, H. Brand, C. E. Düllmann, J. Khuyagbaatar, J. Krier, M. Schädel, T. Torres, and A. Yakushev, J. Radioanal. Nucl. Chem. 299, 1073 (2014).

[15] H. Folger, W. Hartmann, F. P. Heßberger, S. Hofmann, J. Klemm, G. Münzenberg, V. Ninov, K.-H. Schmidt, H.-J. Schött, W. Thalheimer, and P. Armbruster, Nucl. Instrum. Methods Res. Sect., A 334, 69 (1993).

[16] M. Sohani and Y. W. Wilschut, Nucl. Instrum. Methods Res. Sect., A 679, 25 (2012).

[17] A. Ghiorso, J. M. Nitschke, J. R. Alonso, C. T. Alonso, M. Nurmia, G. T. Seaborg, E. K. Hulet, and R. W. Lougheed, Phys. Rev. Lett. 33, 1490 (1974).

[18] T. Ślézak, J. Zmywaczyk, and P. Koniorczyk, AIP Conf. Proc. 2170, 020019 (2019).

[19] IAEA Report No IAEA-TECDOC-1496, 2006.

[20] L. Vlahovic, D. Staicu, A. Küst, and R. J. M. Konings, J. Nucl. Mater. 499, 504 (2018).

[21] W. C. Corwin, Nucl. Instrum. Methods Phys. Res. 136, 41 (1976).

[22] H. Y. Wong, Handbook of Essential Formulae and Data on Heat Transfer for Engineers (Longman, London and New York, 1977).

[23] J. H. Lienhard IV and J. H. Lienhard V, A Heat Transfer Textbook (Phlogiston Press, Cambridge, Mass., 2008).

[24] Ti thermal conductivity, https://www.efunda.com, accessed: 2021-04-28.

[25] S. G. Popov, J. J. Carbajo, V. K. Ivanov, and G. L. Yoder, Oak Ridge National Laboratory Report No ORNL/ TM-2000/351, 2000.

[26] W. C. Michels and S. Wilford, J. Appl. Phys. 20, 1223 (1949).

[27] W. Landensperger and D. Stark, Z. Phys. 180, 178 (1964).

[28] A. V. Arutyunov, S. N. Banchila, and L. P. Filippov, High Temp. 9, 487 (1971).

[29] V. E. Peletsky and V. P. Druzhinin, TBT 11, 212 (1973), http://mi.mathnet.ru/tvt9819.

[30] P.-F. Paradis and W.-K. Rhim, J. Chem. Thermodyn. 32, 123 (2000).

[31] G. Teodorescu, Ph.D. Thesis, Auburn University, Alabama, 2007.

[32] Ti emissivity, https://www.engineeringtoolbox.com, accessed: 2021-04-28.

[33] J. H. Harding, D. G. Martin, and P. E. Potter, Thermophysical and thermochemical properties of fast reactor materials, Harwell Laboratory UKAEA Report EUR 12402, 1989.

[34] Thermophysical Properties of Materials For Nuclear Engineering: A Tutorial and Collection of Data (IAEA, Vienna, 2008), https:/www.iaea.org/publications/7965/.
[35] S. Song and M. M. Yovanovich, in Proceedings of the Twenty-fourth National Heat Transfer Conference and Exhibition, Pittsburgh, 1987 (American Society of Mechanical Engineers, New York, 1987), p. 107.

[36] K. L. Day, in Interstellar Dust and Related Topics, edited by J.M. Greenberg and H. C. Van De Hulst (Springer, Dordrecht, 1973), p. 311.

[37] I. Yasumoto, J. Phys. Chem. 91, 4298 (1987).

[38] O. Leroy, J. Perrin, J. Jolly, M. Péalat, and M. Lefebvre, J. Phys. D 30, 499 (1997).

[39] S. Vanapalli, B. Colijn, C. Vermeer, H. Holland, T. Tirolien, and H. J. M. ter Brake, in 25th International Cryogenic Engineering Conference and the International Cryogenic Materials Conference, 2014; Phys. Procedia 67, 1206 (2015).

[40] F. W. Burkholder, Ph.D. Thesis, University of Colorado, Colorado, 2011.

[41] W. B. Mann, Proc. R. Soc. A 859, 776 (1934).

[42] H. S. Gregory, Proc. R. Soc. A 866, 35 (1935).

[43] B. W. Burslow, Ph.D. Thesis, Imperial College of Science and Technology, London, 1952.

[44] W. M. Trott, D. J. Rader, J. N. Castañeda, J. R. Torczynski, and M. A. Gallis, AIP Conf. Proc. 1084, 621 (2008).

[45] D. J. Rader, W. M. Trott, J. R. Torczynski, J. N. Castañeda, and T.W. Grasser, Measurements of thermal accomodation coefficients, Sandia Report, Technical Report No. SAND2005-6084, 2005.

[46] C. H. Cho, T. K. Ghosh, R. V. Tompson, and S. K. Loyalka, Transaction of the American Nuclear Society and Embedded Topical Meetings and Decommissioning and Spent-Fuel Management and Risk Management-Now More than Ever, San Diego, California, 2003; T. Am. Nucl. Soc. 88, 228 (2003), https://www.researchgate.net/ publication/281826840.

[47] H. Yamaguchi, M. T. Ho, Y. Matsuda, T. Niimi, and I. Graur, Int. J. Heat Mass Transfer 108, 1527 (2017).

[48] IAEA Report No IAEA-TECDOC-949, 1997.

[49] M. Knudsen, Kinetic Theory of Gases (John Wiley and Sons, New York, 1950).

[50] Handbook of Heat Transfer, 3rd ed., edited by W. M. Rohsenow, J. P. Hartnett, and Y. I. Cho (McGraw-Hill, New York, 1998).

[51] B. P. Jelle, A. Gustavsen, and R. Baetens, J. Build. Phys. 34, 99 (2010).

[52] H. J. M. Hanley, R. D. McCarty, and H. Intemann, J. Res. NBS A Phys. Ch. 74A, 331 (1970).

[53] J. F. Ziegler, SRIM - The Stopping and Range of Ions in Matter, available at http://www.srim.org, accessed: 202104-28. 